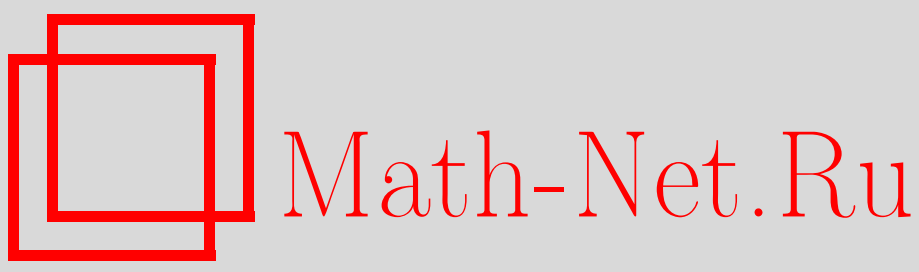

В. Е. Назайкинский, Б.-В. Шульц, Б. Ю. Стернин, Индекс интегральных операторов Фурье на многообразиях с коническими особенностями, Изв. РАН. Сер. матем., 2001, том 65, выпуск 2, 127-154

DOI: https://doi.org/10.4213/im329

Использование Общероссийского математического портала Math-Net.Ru подразумевает, что вы прочитали и согласны с пользовательским соглашением

http: //www.mathnet.ru/rus/agreement

Параметры загрузки:

IP : 54.205 .225 .156

26 апреля 2023 г., $13: 28: 38$ 
УДК 517.9

В. Е. Назайкинский, Б. Ю. Стернин, Б.-В. Шульце

\title{
Индекс интегральных операторов Фурье на многообразиях с коническими особенностями
}

\begin{abstract}
Дается описание однородных канонических преобразований кокасательного расслоения над многообразием с коническими особыми точками. Вычисляется индекс эллиптического интегрального оператора Фурье, полученного квантованием такого преобразования. Ответ дается в терминах индекса эллиптического интегрального оператора Фурье на гладком многообразии и вычетов конормального символа.
\end{abstract}

Библиография: 23 наименования.

\section{Введение}

Интегральньй оператор Фурье (ИОФ), действующий в пространствах функций на гладких компактных многообразиях, называется эллиптическим, если он ассоциирован с некоторым однородным каноническим преобразованием кокасательного расслоения первого многообразия в кокасательное расслоение второго и если его главный символ (амплитуда) всюду отличен от нуля вне нулевого сечения. Эллиптический ИОФ всегда фредгольмов в соответствуюших пространствах Соболева, и потому естественно возникает задача о вычислении его индекса, который является гомотопическим инвариантом канонического преобразования и главного символа. Задача о вычислении индекса ИОФ в классических терминах была поставлена А. Вайнстейном [1] в 1976 г. (в связи с одной задачей, связанной со спектрами римановых многообразий) для операторов с унитарнылм главным символом. Для таких операторов, которые мы будем называть квантованными каноническими преобразованиями, индекс не зависит от конкретного выбора главного символа, если размерность многообразий, на которых действуют операторы, не меньше 3 или если индекс Маслова на графике соответствующего канонического преобразования тривиален по модулю 4 и представляет собой инвариант самого́ канонического преобразования. В то время, когда эта задача ставилась, не было известно (и, по-видимому, неизвестно до сих пор), существуют ли квантованные канонические преобразования с ненулевым индексом. В последние годы усилиями ряда авторов (Эпштейн, Мельроуз, Зелдич, Гийемин и др.) задача о вычислении индекса эллиптических ИОФ была включена в более широкую задачу о вычислении индекса операторов, получаемых квантованием контактных преобразований. Эти преобразования действуют между произвольными компактными контактными многообразиями. Первоначальная постановка задачи, принадлежащая Вайнстейну, соответствует случаю, в котором указанные контактные многообразия являются ко-

Работа выполнена при финансовой поддержке Российского фонда фундаментальных исследований (гранты № 99-01-01100 и 00-01-00161).

(C) В.Е. НАЗАЙКИНСКИй, Б. Ю. СтЕРнин, Б.-В. ШульцЕ, 2001 
сферическими расслоениями многообразий, на которых задан ИОФ, соответствующий некоторому однородному каноническому преобразованию. При этом контактное преобразование получается из однородного канонического стандартным образом (см., например, [2]), а эквивалентность (подобие) ИОФ и квантованного контактного преобразования устанавливается так называемым преобразованием Гийемина [3], [4]. Подробное описание задачи об индексе ИОФ и возможных подходов к ее решению может быть найдено в [5], где, в частности, отмечается, что вычисление индекса произвольных эллиптических ИОФ (а не только квантованных канонических преобразований) представляет наибольший интерес. В недавно появившейся статье [6] Эпштейн и Мельроуз решили проблему Вайнстейна в широкой постановке (т.е. для квантованных контактных преобразований), но лишь в частном случае контактного преобразования, отображающего контактное многообразие в себя. (Для ИОФ это отвечает квантованным каноническим преобразованиям, действуюшим между пространствами функций на одном и том же компактном многообразии.) В этой ситуации в [6] было показано, что индекс квантованного контактного преобразования равен так называемой контактной степени [6] преобразования, которая, в свою очередь, может быть выражена как индекс оператора Дирака, ассоциированного со $\operatorname{spin}^{C}$-структурой на торе контактного преобразования.

Настояшая работа посвящена нахождению формулы индекса эллиптических ИОФ в ситуации, когда конфигурационное пространство имеет особенности конического типа. Многообразия с коническими особенностями занимают в эллиптической теории важное место, и поэтому изучение проблемы индекса ИОФ на таких многообразиях, несомненно, представляет интерес. Полученная нами формула индекса выражается в терминах индекса некоторого вспомогательного ИОФ на гладком компактном многообразии и некоторого (явно выписываемого) инварианта конормального символа (см., например, [7]) исходного ИОФ.

Для случая квантованных канонических преобразований кокасательного расслоения многообразия с коническими особенностями индекс вспомогательного ИОФ может быть вычислен по формуле Эпштейна-Мельроуза из [6]. В обшем случае формула приобретает окончательный вид после того, как в полном объеме будет решена проблема Вайнстейна для гладких многообразий ${ }^{1}$.

Авторы выражают признательность А. Вайнстейну за интерес к настоящей работе и В.Е. Шаталову за ряд полезных дискуссий по вопросам, затронутым в настоящей статье.

Предварительная версия работы опубликована в препринте [8], краткое изложение - в заметке [9].

\section{§1. Квантование канонических преобразований}

1.1. Однородные канонические преобразования. Пусть $M$ - гладкое замкнутое многообразие без края с коническими особыми точками $\left\{\alpha_{1}, \ldots, \alpha_{N}\right\}$ (см., например, [7], [10]). По определению это означает, что $M$ - топологическое пространство, $\stackrel{\circ}{M}=M \backslash\left\{\alpha_{1}, \ldots, \alpha_{N}\right\}-($ открытое) гладкое многообразие, а в окрест-

\footnotetext{
${ }^{1}$ За то время, пока статья находилась в редакции, Е. Лайштнам, Р. Нест и Б. Циган дали полное решение проблемы Вайнстейна для гладких многообразий.
} 
ности каждой особой точки $\alpha_{j}$ многообразие представлено в виде конуса

$$
K_{j}=\left([0,1) \times \Omega_{j}\right) /\left(\{0\} \times \Omega_{j}\right)
$$

где $\Omega_{j}$ - база конуса - гладкое компактное многообразие. В такой окрестности будут использоваться два вида координат, а именно "конические координаты" $(\sigma, \omega)$, $\sigma \in[0,1), \omega \in \Omega_{j}$, и “цилиндрические координаты" $(t, \omega), t \in(0,+\infty], \omega \in \Omega_{j}$, связанные с коническими координатами заменой переменных $r=e^{-t}$. В последнем случае будем также говорить, что $\stackrel{\circ}{M}$ (или $M$ ) представлено как "многообразие с цилиндрическими концами". Допуская некоторую вольность в обозначениях, будем обозначать буквой $\omega$ как точку многообразия $\Omega_{j}$, так и ее локальные координаты на $\Omega_{j}$. Для удобства объединим все точки $\left\{\alpha_{1}, \ldots, \alpha_{N}\right\}$ в одну точку $\alpha_{0}$. При этом база конуса $\Omega$ будет не связной, если $N>1: \Omega=\Omega_{1} \cup \cdots \cup \Omega_{N}$ (несвязное объединение).

Пусть $T^{*} M$ - кокасательное расслоение многообразия $M$. Оно строится следуюшим образом (см., например, [11]). Пусть $M^{\wedge}$ - многообразие с краем, полученное раздутием ("blow-up") конической точки $\alpha$. Раздутие сводится к замене конуса

$$
K=\{\Omega \times[0,1)\} /\{(\Omega \times\{0\})\}
$$

в особой точке $\alpha$ на полуцилиндр

$$
K^{\wedge}=\Omega \times[0,1)
$$

Таким образом, $\partial M^{\wedge}=\Omega$. Для многообразия $M^{\wedge}$ рассмотрим "сжатое кокасательное расслоение" $\widetilde{T}^{*} M^{\wedge}$ (compressed cotangent bundle, в терминологии [12]). По определению $\widetilde{T}^{*} M^{\wedge}$ есть векторное расслоение над $M^{\wedge}$, пространство гладких сечений которого совпадает с

$$
\operatorname{Hom}_{C^{\infty}\left(M^{\wedge}\right)}\left(\operatorname{Vect}_{0}\left(M^{\wedge}\right), C^{\infty}\left(M^{\wedge}\right)\right)
$$

где $\operatorname{Vect}_{0}\left(M^{\wedge}\right)-C^{\infty}\left(M^{\wedge}\right)$-модуль векторных полей на многообразии $M^{\wedge}$, всюду касательных к краю $\partial M^{\wedge}$.

По определению полагают

$$
T^{*} M \stackrel{\text { def }}{\equiv} \widetilde{T}^{*} M^{\wedge} \text {. }
$$

Таким образом, кокасательное расслоение $T^{*} M$ есть многообразие с краем

$$
\partial T^{*} M \cong T^{*} \Omega \times \mathbb{R}
$$

где $T^{*} \Omega$ - обычное кокасательное расслоение к замкнутому многообразию $\Omega$, а сомножитель $\mathbb{R}$ отвечает конормальной переменной $p$. Многообразие $T^{*} M$ снабжено естественной симплектической формой, имеющей особенность на краю. В конических координатах эта форма имеет вид

$$
\omega^{2}=-\frac{1}{r} d p \wedge d r+\omega_{T^{*} \Omega}^{2}
$$


где $\omega_{T^{*} \Omega}^{2}-$ стандартная симплектическая структура на $T^{*} \Omega$. Для дальнейшего полезно записать эту форму и в цилиндрических координатах. В этих координатах пространство $T^{*} M$ выглядит как обычное кокасательное расслоение, к которому добавлен слой над "базой конуса в бесконечности" $\{t=\infty\} \times \Omega$. Симплектическая форма в этих координатах имеет хорошо знакомое выражение

$$
\omega^{2}=d p \wedge d t+\omega_{T^{*} \Omega}^{2}
$$

Через $T_{0}^{*} M=T^{*} M \backslash\{0\}$ обозначим кокасательное расслоение над $M$ с выброшенным нулевым сечением. В слоях расслоения $T_{0}^{*} M$ естественным образом действует группа $\mathbb{R}_{+}$положительных чисел (умножением вектора на число).

ОПРЕДЕЛЕНИЕ 1.1. Однородным Каноническим преобразованием пространства $T_{0}^{*} M$ называется гладкий вплоть до границы диффеоморфизм

$$
g: T_{0}^{*} M \rightarrow T_{0}^{*} M
$$

сохраняющий симплектическую форму на $T_{0}^{*} M$ и коммутирующий с действием группы $\mathbb{R}_{+}$.

Опишем однородные канонические преобразования с помошюю производящих функций.

Пусть $g: T_{0}^{*} M \rightarrow T_{0}^{*} M-$ однородное каноническое преобразование.

График преобразования $g$ является лагранжевым многообразиемв $T_{0}^{*} M \times T_{0}^{*} M$ относительно симплектической формы $\pi_{2}^{*} \omega^{2}-\pi_{1}^{*} \omega^{2}$, где $\pi_{1}$ и $\pi_{2}-$ проекции на первый и второй сомножители в прямом произведении $T_{0}^{*} M \times T_{0}^{*} M$, и во внутpенних точках (т.е. вне границы $\left.\partial T_{0}^{*} M\right)$ описание преобразования $g$ с помощью производящих функций стандартно (см. [2] или, применительно к случаю однородных преобразований, также [13]). Выясним, как устроено преобразование вблизи $\partial T_{0}^{*} M$. Пусть $(\omega, q)$ - канонические координаты на $T^{*} \Omega$. Тогда $(r, \omega, p, q)$ будем называть каноническими координатами на $T^{*} K$ (или, эквивалентно, на $T^{*} \Omega$ вблизи $\left.\partial T^{*} \Omega\right)$.

Пусть $(\rho, \psi, \xi, \eta)$ - канонические координаты на втором экземпляре $T^{*} K$. Преобразование $g$ задается в окрестности гранищы формулами

$$
\begin{array}{ll}
\rho=r \rho_{1}(r, \omega, p, q), & \psi=\psi(r, \omega, p, q), \\
\xi=\xi(r, \omega, p, q), & \eta=\eta(r, \omega, p, q),
\end{array}
$$

где $\rho_{1}, \psi, \xi, \eta$ - гладкие функции, причем $\rho_{1}(0, \omega, p, q) \neq 0$ (так как $g$ - диффеоморфизм).

В цилиндрических координатах $(\tau=-\ln \rho, t=-\ln r)$ эти форомулы приобретают вид

$$
\begin{array}{ll}
\tau=t+\xi\left(e^{-t}, \omega, p, q\right), & \psi=\psi\left(e^{-t}, \omega, p, q\right) \\
\xi=\xi\left(e^{-t}, \omega, p, q\right), & \eta=\eta\left(e^{-t}, \omega, p, q\right)
\end{array}
$$

Доказательство следующей леммы, которое было проведено в [11] для неоднородного случая, является чисто техническим, и мы его опускаем. 
Лемма 1.1. а) Отображение

$$
g(p): \begin{cases}T^{*} \Omega \rightarrow T^{*} \Omega, & p \neq 0 \\ T_{0}^{*} \Omega \rightarrow T_{0}^{*} \Omega, & p=0\end{cases}
$$

задаваемое формулами

$$
\psi=\psi(0, \omega, p, q), \quad \eta=\eta(0, \omega, p, q),
$$

является симплектоморфизмом при всех р.

б) $\xi(0, \omega, p, q) \equiv 0$.

Теперь можем сформулировать лемму о локальных координатах.

ЛЕмма 1.2. Пусть $\left(0, \psi_{0}, \xi_{0}, \eta_{0}\right)=g\left(0, \omega_{0}, p_{0}, q_{0}\right)$. Тогда существует подмножество $I \subset\{1, \ldots, n-1\}$ такое, что функиии $\left(\rho, \psi_{I}, \eta_{\bar{I}}, p, q\right)$, где $\bar{I}=$ $\{1, \ldots, n-1\} \backslash I$, образуют систему локальных координат на графике преобразования $g$ в окрестности точки

$$
z_{0}=\left(0, \omega_{0}, p_{0}, q_{0}, 0, \psi_{0}, \xi_{0}, \eta_{0}\right) \in \operatorname{graph} g \subset T^{*} K \times T^{*} K .
$$

ДокАЗАТЕЛЬСтво. Дифференциалы $d \rho, d p$ и $d q$ линейно независимы на $L=$ graph $g$ в точке $z_{0}$, так как при $r=0$ имеем $d \rho=\rho_{1} d r, \rho_{1} \neq 0$, a $(r, p, q)$ - независимые координаты. Пусть $I \subset\{1, \ldots, n-1\}$ - максимальное подмножество такое, что дифференциалы $\left(d \rho, d \psi_{I}, d p, d q\right)$ независимы на $L$ в точке $z_{0}$ (здесь $n=\operatorname{dim} M$ ). Тогда система $\left(\rho, \psi_{i}, \eta_{\bar{I}}, p, q\right)$ искомая. Это вытекает из обычной леммы о локальных координатах (см., например, [14]), примененной к каноническому преобразованию $g\left(p_{0}\right)$.

Таким образом, график преобразования $g$ может быть покрыт конечным числом канонических карт (поскольку мы имеем дело с однородным случаем, карты будут $\mathbb{R}_{+}$-инвариантны). В этих картах преобразование определяется производящими функциями вида

$$
S_{I}\left(\tau, \psi_{I}, \eta_{\bar{I}}, p, q\right)=p \tau+S_{1 I}\left(e^{-\tau}, \psi_{I}, \eta_{\bar{I}}, p, q\right) .
$$

Здесь $I \subset\{1, \ldots, n-1\}$ - тип карты, $(\tau, \psi)$ и $(t, \omega)$ - координаты на втором и первом экземплярах многообразия $M$ соответственно, а $p, q$ - координаты, двойственные к $(\tau, \psi)$ (в частности, переменная $p$ - аргумент конормального символа). В такой карте преобразование

$$
g:(t, \omega, p, q) \mapsto(\tau, \psi, \xi, \eta)
$$

задается обычными формулами

$$
\begin{aligned}
t & =\frac{\partial S_{1}}{\partial p}, \quad \xi=\frac{\partial S_{1}}{\partial \tau}, \\
\omega & =\frac{\partial S_{1}}{\partial q}, \quad \eta_{I}=\frac{\partial S_{1}}{\partial \psi_{I}}, \quad \psi_{\bar{I}}=\frac{\partial S_{1}}{\partial \eta_{\bar{I}}} .
\end{aligned}
$$

Поскольку преобразование однородно, отсюда следует, что производящие функции первого порядка однородны по импульсным переменным, т. е.

$$
S_{1 I}\left(e^{-\tau}, \psi_{I}, \lambda \eta_{\bar{I}}, \lambda p, \lambda q\right)=\lambda S_{1 I}\left(e^{-\tau}, \psi_{I}, \eta_{\bar{I}}, p, q\right)
$$

при $\lambda>0$. 
1.2. Интегральные операторы Фурье. Здесь мы опишем процедуру квантования однородных канонических преобразований. Именно, сопоставим каждому однородному каноническому преобразованию

$$
g: T_{0}^{*} M \rightarrow T_{0}^{*} M
$$

некоторый класс интегральных операторов Фурье $T(g, a)$ (см. ниже (2)) в весовых пространствах Соболева $H_{\gamma}^{s}(M)$.

Напомним определение весовых пространств Соболева на многообразии с коническими особенностями (см., например, [7], [10]). Пусть $M$ - многообразие с коническими особенностями $\left\{\alpha_{1}, \ldots, \alpha_{N}\right\}$. Пусть также $\gamma_{1}, \ldots, \gamma_{N} \in \mathbb{R}$ - заданные числа (весовые показатели). Рассмотрим разбиение единицы

$$
1=\sum_{j=0}^{N} e_{j}(x), \quad x \in M,
$$

где $e_{0}(x)=0$ в окрестности особенностей, а $e_{j}(x), j=1, \ldots, N$, имеет носитель в малой окрестности точки $\alpha_{j}$, в которой действуют конические координаты. Для функции $u(x)$ на $M$ полагаем

$$
\|u\|_{s, \gamma_{1}, \ldots, \gamma_{N}}^{2}=\left\|e_{0} u\right\|_{s}^{2}+\sum_{j=1}^{N}\left\|e_{j} u\right\|_{s, \gamma_{j}}^{2},
$$

где $\left\|e_{0} u\right\|_{s}$ - обычная соболевская норма в гладкой части многообразия $M$, а $\left\|e_{j} u\right\|_{s, \gamma_{j}}$ задается в конических координатах формулой

$$
\left\|e_{j} u\right\|_{s, \gamma_{j}}^{2}=\int_{0}^{\infty}\left\|\left(1+\left(i r \frac{\partial}{\partial r}\right)^{2}+\Delta_{\Omega_{j}}\right)^{3 / 2}\left(r^{-\gamma_{j}} e_{j} u\right)\right\|^{2} \frac{d r}{r},
$$

где $\Delta_{\Omega_{j}}$ - (положительный) оператор Лапласа-Бельтрами на $\Omega_{j}$ относительно какой-либо римановой метрики.

Пространство $H_{\gamma_{1}, \ldots, \gamma_{N}}^{s}(M)$ является пополнением пространства $C_{0}^{\infty}(M \backslash$ $\left.\left\{\alpha_{1}, \ldots, \alpha_{N}\right\}\right)$ по норме (1.1). Пространства $H_{\gamma_{1}, \ldots, \gamma_{N}}^{s}(M, E)$, где $E$ - расслоение над $M$, вводятся аналогичным образом.

Мы объединим все конические точки многообразия $M$ в одну точку $\alpha$. Поэтому в дальнейшем считаем, что $\gamma_{1}=\cdots=\gamma_{N}=\gamma$ и соответствуюшее пространство Соболева обозначим через $H_{\gamma}^{s}(M)$. Переход к общему случаю произвольных $\gamma_{1}, \ldots, \gamma_{N}$ не представляет трудностей.

Вначале рассмотрим случай $\gamma=0$ (при этом в цилиндрических координатах мы имеем дело с обычными пространствами Соболева на цилиндре). Напомним, что коническая точка соответствует точке $t=\infty$. Значения $t \in(-\infty, 0]$ являются, следовательно, несущественными, и поэтому некоторые из последующих формул должны, в действительности, содержать срезающие функции, обрашаюшиеся в нуль при $t<0$. Мы будем опускать рассмотрения, связанные с этими срезающими функциями.

Пусть

$$
L_{g}=\left\{\left(\beta, \widetilde{\beta} \in T_{0}^{*} M \mid \widetilde{\beta}=g(\beta)\right\} \subset T_{0}^{*} M \times T_{0}^{*} M\right.
$$


- график преобразования $g$. Многообразие $L_{g}$ является лагранжевым в $T_{0}^{*} M \times$ $T_{0}^{*} M$ относительно симплектической структуры

$$
\widetilde{\omega}^{2}=\pi_{2}^{*} \omega^{2}-\pi_{1}^{*} \omega^{2}
$$

где $\pi_{i}^{*}, i=1,2,-$ каноническая проекция многообразия $L_{g}$ на $i$-й сомножитель и $\omega^{2}$ есть стандартная симплектическая структура на $T_{0}^{*} M$. На $L_{g}$ выбираем квантованную меру $\mu[14]$ такую, что $\mu$ является однородной порядка $n$ относительно действия группы $\mathbb{R}_{+}$.

Пусть $\mathscr{K}_{g}$ - канонический оператор Маслова [15] на лагранжевом многообразии $L_{g}$ с мерой $\mu$ в его однородном варианте (см. [14], [16]). Канонический оператор действует в пространствах

$$
\mathscr{K}_{g}: \mathscr{O}\left(L_{g}\right) \rightarrow \mathscr{D}^{\prime}(M \times M)
$$

где $\mathscr{O}\left(L_{g}\right)=\bigcup_{m} \mathscr{O}^{m}\left(L_{g}\right)$ - пространство асимптотически $\mathbb{R}_{+}$-однородных функций на $L_{g}$ (соответственно $\mathscr{O}^{m}\left(L_{g}\right)$ - пространство асимптотически однородных функций степени не вьше $m)$ и $\mathscr{D}^{\prime}(M \times M)$ - пространство распределений на $M \times M$. Явное выражение для канонического оператора может быть найдено в цитированных выше работах.

Пусть теперь $a \in \mathscr{O}\left(T_{0}^{*} M\right)$. Обозначим через $T(g, a)$ интегральный оператор с ядром Шварца ${ }^{2}$

$$
\mathscr{K}_{g}\left[\pi_{1}^{*} a\right](x, y), \quad x, y \in M .
$$

Заметим, что канонический оператор $\mathscr{K}_{g}$ и, следовательно, оператор $T(g, a)$ зависят от выбора квантованной меры $\mu$ (даже в главном члене).

Teоpema 1.1. Пусть $a \in \mathscr{O}^{m}\left(T_{0}^{*} M\right)$. Тогда оператор $T(g, a)$ непрерывен в пространствах

$$
T(g, a): H_{0}^{s}(M) \rightarrow H_{0}^{s-m}(M)
$$

для любого $s \in \mathbb{R}$.

Эта теорема вытекает из следующей ниже леммы. (Использованный здесь метод является стандартным в доказательстве утверждений, аналогичных данной теореме.) Обозначим для краткости оператор (1.2) через $T$. Пусть $T^{*}-$ сопряженньй оператор для $T$ относительно скалярного произведения в $L^{2}$ (мера вблизи конической точки есть $d t d \omega)$.

ЛЕмма 1.3. Оператор $T^{*} T$ является коническим псевдодифференииальныцм оператором [17] порядка $2 m$ в шкале $H_{0}^{s}(M)$ с главным символом $|a|^{2}\left|\pi_{1}^{-1 *}(\mu) / \mu_{0}\right|$. Здесь

$$
\mu_{0}=\frac{1}{n !}\left(\omega^{2}\right)^{\wedge n}
$$

- стандартная форма облема на $T_{0}^{*} M$.

Доказательство будет дано в приложении.

\footnotetext{
${ }^{2}$ Предполагается, что на $M$ зафиксирована некоторая форма объема, в окрестности конической точки имеющая вид $d t \wedge v$, где $v$ - не зависящая от $t$ форма объема на $\Omega$; это позволяет рассматривать ядра Шварца как функции, а не плотности.
} 
Рассмотрим теперь случай $\gamma \neq 0$. Для того чтобы определить оператор $T_{\gamma}=$ $T(g, a)$ в шкале $H_{\gamma}^{s}(M)$, требуется, чтобы все функции, входящие в определение локальных канонических операторов в окрестности особой точки, были аналитичны по переменной $p$ в некоторой окрестности весовой линии

$$
\mathscr{L}_{\gamma}=\{\operatorname{Im} p=\gamma\} .
$$

Если это не так, необходимо модифицировать определение локальных канонических операторов. Именно, все однородные функции, входящие в определение (фаза, амплитуда, плотность меры, элемент разбиения единицы), заменяются на асимптотически однородные функции переменных $\left(p, q, \eta_{\bar{I}}\right)$ с теми же самыми асимптотиками на бесконечности и со следующим свойством: все новые функции являются гладкими везде (включая точку $\left.\left(p, q, \eta_{\bar{I}}\right)=(0,0,0)\right)$ и допускают аналитическое продолжение относительно переменной $p$ в некоторую полосу $|\operatorname{Im} p|\langle K \mathrm{c} K\rangle|\gamma|$, $K$, здесь и ниже, есть некоторое положительное число. Более того, аналитическое продолжение должно быть также асимптотически однородным в этой полосе.

Это может быть сделано многими различными способами, например сверткой преобразования Фурье гладкой функции с компактным носителем и единичным интегралом (см., например, [18]); такой способ дает целую функцию от $p$.

Таким образом, интегральные операторы $T(g, a)$ корректно определены в весовых соболевских пространствах с ненулевым показателем $\gamma$. Следующий результат является прямым обобшением теоремы 1.1 и леммы 1.3 и может быть выведен из них.

Teорема 1.2. Пусть $g: T_{0}^{*} M \rightarrow T_{0}^{*} M-$ однородное каноническое преобразование, $a \in \mathscr{O}^{m}\left(T_{0}^{*} M\right)$ и все әлементы в конструкции канонического оператора $\mathscr{K}_{g}$ являются аналитическими по $р$ в полосе $|\operatorname{Im} p|<K$ и удовлетворяют перечисленным выше условиям. Тогда интегральный оператор $T \equiv T_{\gamma} \equiv T_{\gamma}(g, a)$ непрерывен в весовых пространствах Соболева

$$
T: H_{\gamma}^{s}(M) \rightarrow H_{\gamma}^{s-m}(M)
$$

$\partial \Omega s s \in \mathbb{R} u|\gamma|<K$.

Более того, оператор $T^{*} T$, где $T^{*}$ - сопряженный оператор $к$ оператору $T$ относительно скалярного произведения $L_{\gamma}^{2}(M) \equiv H_{\gamma}^{0}(M)$, является псевдодифференциальным оператором порядка $2 m$ в шкале $\left\{H_{\gamma}^{s}(M)\right\} \quad c|\gamma|<K$. Главный символ оператора $T^{*} T$ есть $|a|^{2}\left|\pi_{1}^{-1 *}(\mu) / \mu_{0}\right|$ (здесь мера $\mu_{0}$ та жее самая, что и в лемме 1.3).

Причина использования скалярного произведения в пространстве $L_{\gamma}^{2}(M)$ для определения сопряженного оператора состоит в том, что при этом определении полный символ оператора $T^{*} T$ является аналитической функцией переменной $p=$ $\operatorname{Re} p+i \gamma$.

Мы определим конормальный символ $T(p)$ оператора $T=T(g, a)$ следующим образом (см. [11]). Пусть $g(p)$ - семейство канонических преобразований пространств $T_{0}^{*} \Omega$, введенное в лемме 1.1, а) (и получающееся из $g$ ограничением на "слой" над конической точкой $r=0)$. Далее, пусть $a(p), p \in \mathbb{C},-$ семейство функций на $T^{*} \Omega$, заданное формулой

$$
a(p)(\omega, q)=\left.a(r, p, \omega, q)\right|_{r=0}, \quad(\omega, q) \in T^{*} \Omega .
$$


Мы полагаем

$$
T(p)=T(g(p), a(p))
$$

где в правой части равенства стоит семейство интегральных операторов Фурье с амплитудой $a(p)$, ассоциированных с каноническими преобразованиями $g(p)$, причем подразумевается, что использованная в этом определении мера $d \mu(p)$ удовлетворяет условию

$$
d \mu=d \mu(p) \wedge \frac{d r}{r}
$$

а элементы разбиения единищы получаются из соответствующих элементов для $T(p)$ ограничением в слой над точкой $r=0$. Заметим, что при таком определении конормального символа имеет место равенство

$$
\mathscr{M}^{-1} T(p) \mathscr{M}=s-\lim _{\lambda \rightarrow 0} U_{\lambda} T(g, a) U_{\lambda}^{-1}
$$

где $\mathscr{M}$ - преобразование Меллина, а $U_{\lambda}$ - растяжение по $r$ :

$$
U_{\lambda} \varphi(\omega, r)=\varphi(\omega, \lambda r)
$$

Заметим также, что зависимость конормального символа $T(p)$ от переменной $p$ в комплексной области определяется выбором аналитического сглаживания (впоследствии этот выбор предполагается фиксированным).

1.3. Квантованные канонические преобразования. Квантованные канонические преобразования (см., например, [5], [6] и приведенную в этих работах библиограбию, где они определены для гладкого случая с помощью проекторов Сегё и преобразования Гийемина и назьваются квантованными контактными преобразованиями) являются специальным случаем общих интегральных операторов Фурье (1.2). Именно, надо положить амплитуду $а$ равной 1 и взять на лагранжевом многообразии $L_{g}$ меру, соответствуюшую некоторой комплексной поляризации на $T_{0}^{*} M$. (Таким образом, для различных поляризаций получаются различные квантованные контактные преобразования $T(g, 1)$. Однако индекс операторов $T(g, 1)$ не зависит от выбора поляризации [6].) Для случая многообразий с коническими особенностями поляризация вблизи конической точки должна быть выбрана так, чтобы символ имел подходящее поведение при $t \rightarrow \infty(t-$ цилиндрическая координата). Например, всегда допустима поляризация, не зависящая от $t$ в цилиндрических координатах вблизи конической точки.

Будем обозначать квантованное каноническое преобразование, соответствующее отображению $g$, через $T_{\gamma}=T_{\gamma}(g)=T(g)$, т. е. мы явно не указываем поляризацию. В случае многообразий с коническими особенностями нужно иметь в виду, что имеется дополнительная неоднозначность в определении оператора $T(g)$ по модулю компактных операторов (конормальньй символ зависит от выбора аналитического сглаживания в конструкции канонического оператора).

Для квантованных канонических преобразований теорема 1.2 выглядит следующим образом. 
Teорема 1.3. Пусть $g: T_{0}^{*} M \rightarrow T_{0}^{*} M$ - однородное каноническое преобразование, и пусть все әлементы, участвующие в построении канонического оператора $\mathscr{K}_{g}$, являются аналитическими по переменной $p$ в полосе $|\operatorname{Im} p|<K$ и удовлетворяют сформулированным выше условиям. Тогда квантованное каноническое преобразование

$$
T(g): H_{\gamma}^{s}(M) \rightarrow H_{\gamma}^{s}(M)
$$

является непрерывным оператором в весовых пространствах Соболева для $s \in \mathbb{R} u|\gamma|<K$.

Более того, оператор $T^{*}(g) T(g)$ является псевдодифференциальным оператором порядка нуль с әллиптическим главным символом в шкале $\left\{H_{\gamma}^{s}(M)\right\}$ $c|\gamma|<K$.

1.4. Эллиптичность и фредгольмовость. Выясним, при каких условиях оператор (1.3) будет фредгольмовым оператором в шкале $\left\{H_{\gamma}^{s}(M)\right\}$ с данным $\gamma$. Пусть символ $a$ формально эллиптический (т. е. отличен от нуля для больших значений $|p|+|q|)$.

Как показано в теореме 1.2 , оператор $T^{*} T$ есть псевдодифференциальный оператор (п. д. о.) с главным символом $|a|^{2}$ и конормальным символом $T^{*}(p) T(p)$. Аналогичное утверждение верно и для оператора $T T^{*}$. Отсюда вытекает в силу эллиптической теории для п. д. о. на многообразиях с коническими особенностями (см., например, [7], [10]), что $T^{*}(p) T(p)$ (а значит, и $T(p)$ ) конечномероморфно обратим в полосе $|\operatorname{Im} p|<K$ и имеет конечное число полюсов в любой полосе $|\operatorname{Im} p|<K-\varepsilon$, $\varepsilon>0$. На любой весовой прямой $\operatorname{Im} p=\gamma$, не содержащей полюсов семейства $T(p)$, оператор $T$ будет фредгольмовым в шкале $\left\{H_{\gamma}^{s}(M)\right\}$. В качестве регуляризатора можно взять $S T^{*}$ или $T^{*} S_{1}$, где $S$ и $S_{1}$ - регуляризаторы для $T^{*} T$ и $T T^{*}$ соответственно.

Таким образом, справедлива

Tеорема 1.4. Oператор $T=T(g, a)$ фредгольмов в шкале $\left\{H_{\gamma}^{s}(M)\right\}$, если выполнены следующие условия:

1) амплитуда а формально әллиптична;

2) конормальный символ $T(p)$ обратим на весовой прямой $\{\operatorname{Im} p=\gamma\}$.

\section{§2. Индекс интегральных операторов Фурье}

В этом параграфе будет получена формула индекса для фредгольмова интегрального оператора Фурье $T(g, a)$ на многообразии $M$ с коническими особенностями при некоторых условиях симметрии. Индекс выражается в терминах конормального символа $T(p)$ и индекса интегрального оператора Фурье на дубле $2 M$. В п. 2.1 строится регуляризатор специального вида и вычисляется изменение индекса при сдвиге весовой прямой ("относительная" теорема об индексе). Этот результат, конечно, не использует никаких условий симметрии. В п. 2.2 вводится условие симметрии и проводится "склейка", которая редуцирует сумму индексов двух операторов на многообразии $M$ к некоторому оператору на дубле $2 M$. В п. 2.3 результаты, полученные в первых двух пунктах, используются для доказательства основной теоремы об индексе.

На протяжении всего этого параграфа предполагается, что символ рассматриваемого оператора $T$ вблизи конической точки является голоморфной функцией 
переменной $p$ в достаточно широкой полосе, содержашей все весовые линии, входяшие в рассмотрение.

2.1. Регуляризатор и формула "относительного индекса". Пусть $T=$ $T(g, a)$ - эллиптический интегральный оператор Фурье на $M$. Предположим, что конические координаты в окрестности особой точки выбраны и забиксированы. Прогомотопируем оператор $T$ в классе фредгольмовых эллиптических интегральных операторов Фурье (ИОФ) к такому оператору (который будем обозначать той же буквой $T$ ), у которого коэффищиенты в окрестности конической точки не зависят от $r$ (или, в цилиндрических координатах, от $t$ при достаточно больших $t$ ). При этом гомотопия выбирается такой, чтобы вдоль нее конормальный символ не менялся. Такая гомотопия может быть построена следующим образом (ср. [19]). В окрестности конической точки представим оператор $T$ в виде

$$
T=T\left(\stackrel{2}{r}, i r \frac{1}{\partial r}\right),
$$

где $T(r, p)$ - операторнозначная функция (ее значения суть ИО $\Phi$ на базе $\Omega$ конуса). Положим, далее,

$$
T(\tau)=T\left(\varphi_{\tau}(\stackrel{2}{r}), i r \frac{\frac{1}{\partial}}{\partial r}\right),
$$

где $\varphi_{\tau}(r)$ - гладкая функция, $\tau \in[0,1]-$ параметр гомотопии, $\varphi_{0}(r)=r$, a $\varphi_{1}(r)$ обладает следующими свойствами:

$$
\begin{array}{lll}
\varphi_{1}(r)=r & \text { при } r>1, \\
\varphi_{1}(r)=0 & \text { при } \quad r<1 / 2 .
\end{array}
$$

Новый оператор $T(1)$ (который мы по-прежнему обозначим через $T$ ) соответствует новому каноническому преобразованию (которое мы по-прежнему обозначаем через $g$ ), гомотопному первоначальному. Вблизи конической точки $t$-компонента уравнений для $g$ в цилиндрических координатах имеет вид

$$
\tau=t+F(\omega, p, q) \equiv t+\frac{\partial S_{1 I}}{\partial p}\left(0, \psi_{I}, \eta_{\bar{I}}, p, q\right)
$$

где $S_{1 I}\left(0, \psi_{I}, \eta_{\bar{I}}, p, q\right)$ - производящая функция конормального семейства в соответствующей канонической карте.

Пусть $R_{0}$ - обратный оператор для $T_{0}$ (индекс 0 означает замораживание коэффициентов при $r=0$, т. е. если вблизи конической точки $T=T\left(r, i r \frac{\partial}{\partial r}\right)$, то $T_{0}=T\left(0, i r \frac{\partial}{\partial r}\right)$ - оператор на конусе $\left.K\right)$. Очевидно, для конормальных символов имеем

$$
R(p)=R_{0}(p)=T_{0}^{-1}(p)=T^{-1}(p),
$$

и функция $R(p)$ имеет полюсы на тех весовых прямых, для которых оператор $T$ не фредгольмов.

Построим также (методами обычной эллиптической теории) интегральный оператор Фурье $R_{1}$ такой, что

$$
T R_{1}=1+Q, \quad R_{1} T=1+Q^{\prime}
$$


где $Q, Q^{\prime}$ - операторы бесконечного отрицательного порядка в шкале $\left\{H_{\gamma}^{s}(M)\right\}$. При этом символы операторов $R_{1}, Q$ и $Q^{\prime}$ аналитичны (вблизи особой точки). Заметим, что отсутствие полюсов достигнуто ценой того, что $R_{1}$ не есть регуляризатор, операторы $Q$ и $Q^{\prime}$, разумеется, не компактны в шкале $\left\{H_{\gamma}^{s}\right\}$.

Далее, пусть $e_{0}=e_{0}(r), e_{1}=1-e_{0}$ - разбиение единицы на $M$ такое, что $e_{0} \equiv 1$ в окрестности особой точки и $e_{0} \equiv 0$ вне большей окрестности, и пусть $\psi_{0}=\psi_{0}(v)$ и $\psi_{1}=\psi_{1}(v), v \in M,-$ такие гладкие функции, что:

1) $\psi_{0} \equiv 0$ вне окрестности конической точки, в которой справедливо "коническое" представление, а $\psi_{1} \equiv 0$ в окрестности конической точки;

2) в окрестности конической точки для достаточно больших значений $\lambda$ справедливо соотношение

$$
\psi_{i}(\lambda v) \equiv 1 \quad \text { на } \quad \operatorname{supp} e_{i}(\lambda v) \cup \operatorname{supp}\left(\pi\left(g\left(\pi^{*} \operatorname{supp} e_{i}(\lambda v)\right)\right)\right)
$$

где $\pi: T_{0}^{*} M \rightarrow M-$ естественная проекция, и действие $\lambda \in \mathbb{R}_{+}$на $v$ корректно определено в конических координатах.

Существование таких функций очевидно, поскольку $r$-компонента преобразования $g$ в конических координатах имеет вид

$$
\widetilde{r}=r f(r, \omega, p, q)
$$

где $f(r, \omega, p, q)>0$ - однородная фуункция нулевого порядка по переменньм $(p, q)$ и, следовательно, ограничена сверху и снизу ненулевыми константами.

Тогда оператор

$$
R=\psi_{0}(\lambda r) R_{0} e_{0}(\lambda r)+\psi_{1}(\lambda r) R_{1} e_{1}(\lambda r)
$$

будет, очевидно, регуляризатором для $T$ при всех достаточно больших $\lambda$.

Индекс ind $\left(T_{\gamma}\right)$ оператора $T$ в шкале $\left\{H_{\gamma}^{s}(M)\right\}$ выражается формулой

$$
\operatorname{ind}\left(T_{\gamma}\right)=\operatorname{ind}\left\{T: H_{\gamma}^{s}(M) \rightarrow H_{\gamma}^{s-m}(M)\right\}=\operatorname{trace}_{\gamma}(1-R T)-\operatorname{trace}_{\gamma}(1-T R)
$$

где $\operatorname{trace}{ }_{\gamma}$ - след оператора в пространстве $L_{\gamma}^{2}(M)$.

Стандартными преобразованиями получаем (индекс $\gamma$ у символа "trace" для краткости опускаем)

$$
\begin{aligned}
\operatorname{ind}\left(T_{\gamma}\right)= & \operatorname{trace}\left(\psi_{1} Q e_{1}\right)-\operatorname{trace}\left(\psi_{1} Q^{\prime} e_{1}\right)-\operatorname{trace}\left(\psi_{0} R_{0}\left[e_{0}, T\right]+\psi_{1} R_{1}\left[e_{1}, T\right]\right) \\
& -\operatorname{trace}\left(\left[\psi_{0}, T\right] R_{0} e_{0}+\left[\psi_{1}, T\right] R_{1} e_{1}\right)
\end{aligned}
$$

где [, ] - коммутатор операторов, или

$$
\operatorname{ind}\left(T_{\gamma}\right)=I_{1}+I_{2}
$$

где $I_{1}$ - сумма первых двух слагаемых, а $I_{2}$ - сумма членов, содержаших коммутаторы.

Воспользуемся теперь следующим утверждением (следствием из теоремы Лидского; см., например, [20]). 
ЛЕмма 2.1. Если $H_{1}, H_{2}$ - гильбертовы пространства и

$$
A: H_{1} \rightarrow H_{2}, \quad B: H_{2} \rightarrow H_{1}
$$

- такие линейные операторы, что оба произведения $А В$ и ВА принадлежсат trace-классу, mo

$$
\operatorname{trace}(A B)=\operatorname{trace}(B A) \text {. }
$$

Пользуясь этой леммой, преобразуем член $I_{2}$ следуюшим образом:

$$
\begin{aligned}
-I_{2}= & \operatorname{trace}\left(R_{0}\left[e_{0}, T\right]+R_{1}\left[e_{1}, T\right]\right)+\operatorname{trace}\left(\left(1-\psi_{0}\right) R_{0}\left[e_{0}, T\right]+\left(1-\psi_{1}\right) R_{1}\left[e_{1}, T\right]\right) \\
& +\operatorname{trace}\left(\left[\psi_{0}, T\right] R_{0} e_{0}\right. \\
= & \operatorname{trace}\left(R_{0}\left[e_{1}, T\right] R_{1} e_{1}\right) \\
& \left.+\operatorname{trace}\left(R_{0} e_{0}\left[\psi_{0}, T\right]+R_{1}, T\right]+R_{0}\left[e_{0}, T\right]\left(1-\psi_{0}\left[\psi_{1}, T\right]\right)+R_{1}\left[e_{1}, T\right]\left(1-\psi_{1}\right)\right) \\
= & \operatorname{trace}\left(R_{0}\left[e_{0}, T\right] \psi_{0}+R_{0} e_{0}\left[\psi_{0}, T\right]+R_{1}\left[e_{1}, T\right] \psi_{1}+R_{1} e_{1}\left[\psi_{1}, T\right]\right) \\
= & \operatorname{trace}\left(R_{0}\left[e_{0} \psi_{0}, T\right]+R_{1}\left[e_{1} \psi_{1}, T\right]\right)=\operatorname{trace}\left\{\left(R_{0}-R_{1}\right)\left[e_{0}, T\right]\right\} .
\end{aligned}
$$

Здесь мы применили лемму 2.1 к подчеркнутым членам и воспользовались равенствами $e_{i} \psi_{i}=e_{i}$ и $e_{1}+e_{2}=1$.

Объясним кратко, почему лемма 2.1 может быть применена к подчеркнутым членам. Рассмотрим, например, член $\left[\psi_{0}, T\right] R_{0} e_{0}$. Нужно показать, что операторы $\left[\psi_{0}, T\right] R_{0} e_{0}$ и $R_{0} e_{0}\left[\psi_{0}, T\right]$ принадлежат trace-классу операторов в $L_{2, \gamma}(M)$. Имеем

$$
\begin{aligned}
S \equiv\left[\psi_{0}, T\right] R_{0} e_{0} & =\left[\psi_{0}-1, T\right] R_{0} e_{0} \\
& =\left(\psi_{0}-1\right) T R_{0} e_{0}+T\left(1-\psi_{0}\right) R_{0} e_{0} .
\end{aligned}
$$

Условие (2.2) означает, что на носителях функций $\psi_{i}$ и $e_{i}$ оба члена в правой части являются операторами с бесконечно гладкими ядрами. Для того чтобы доказать, что они являются операторами trace-класса, необходимо дополнительно изучить, что происходит вблизи конической точки. Мы будем использовать цилиндрическую координату $t$, тогда существенно поведение рассматриваемых объектов вблизи $t=\infty$. Поскольку выражение $\psi_{0}-1$ обрашается в нуль в окрестности бесконечности, можно написать

$$
\psi_{0}-1=e^{-\varepsilon t} \kappa(t)
$$

где функция $\kappa(t)$ ограничена вместе со всеми производными (напомним, что большие отрицательные значения $t$ для нас несущественны). Теперь, используя обычную формулу композиции псевдодифференциальных операторов и интегральных операторов Фурье, получаем

$$
\kappa T R_{0} e_{0}=F,
$$

где $F$ - псевдодифференциальный оператор порядка $-\infty$, символ которого удовлетворяет соответствуюшим оценкам равномерно по $t$; наличие убываюшего множителя $e^{-\varepsilon t}$ гарантирует теперь, что оператор

$$
\left(\psi_{0}-1\right) T R_{0} e_{0}=e^{-\varepsilon t} \kappa T R_{0} e_{0}=e^{-\varepsilon t} F
$$

принадлежит trace-классу. 
Далее имеем

$$
T\left(1-\psi_{0}\right) R_{0} e_{0}=e^{-\varepsilon t}\left(e^{\varepsilon t} T e^{-\varepsilon t}\right) \kappa R_{0} e_{0}=e^{-\varepsilon t} \widetilde{T} \kappa R_{0} e_{0}
$$

где символ оператора $\widetilde{T}$ отличается от символа оператора $T$ на сдвиг $i \varepsilon$ в аргументе $p$ (другими словами, весовая линия для оператора $T$ сдвинута на $i \varepsilon$ ). При достаточно малых $\varepsilon$ новая весовая линия все еще лежит в области аналитичности символа оператора $T$, откуда следует, что оператор $\widetilde{T}$ имеет те же самые свойства, что и оператор $T$. Теперь видно, что оператор $T\left(1-\psi_{0}\right) R_{0} e_{0}$ также принадлежит trace-классу.

Доказательство утверждения для члена $R_{0} e_{0}\left[\psi_{0}, T\right]$ включает также коммутацию экспоненты $e^{-\varepsilon t}$ с оператором $R_{0}$. Последнее не приводит к дополнительным эффектам. (Поскольку оператор $R_{0}$ не имеет полюсов на прямой $\mathscr{L}_{\gamma}$, отсюда следует, с учетом того, что все полюсы лежат в некотором секторе, что оператор $R_{0}$ не имеет полюсов на весовых линиях, достаточно близких к $\mathscr{L}_{\gamma}$.)

Для другого подчеркнутого члена нужные рассуждения аналогичны.

Устремим теперь $\lambda \rightarrow 0$ и регуляризуем интеграл $I_{1}$ по методу работы [21]. Получим

$$
\operatorname{ind}\left(T_{\gamma}\right)=\operatorname{trace}_{\gamma}\left\{\left(R_{0}-R_{10}\right)\left[T_{0}, e_{0}\right]\right\}+\operatorname{trace}_{\gamma} \widetilde{Q},
$$

где $\widetilde{Q}-$ п. д. о. trace-класса с символом, голоморфным по $p$.

Для вычисления первого слагаемого в формуле (2.3) воспользуемся следующей леммой.

Лемма 2.2. Пусть $F(p), G(p)$ - гладкие операторнозначнье функиии, у которых все производные имеют не более чем степенной рост, и пусть $f(t)$ гладкая функиия такая, что $f^{\prime}(t)$ финитна. Тогда

$$
\begin{aligned}
H(p) & \stackrel{\text { def }}{=} \int_{-\infty}^{\infty} \operatorname{Smbl} \underset{-i \frac{\partial}{\partial t}, t, t}{,}\left\{G\left(-i \frac{\partial}{\partial t}\right) \circ\left[F\left(-i \frac{\partial}{\partial t}\right), f(t)\right]\right\} d t \\
& =-i G(p) \frac{\partial F}{\partial p}(p)(f(+\infty)-f(-\infty))
\end{aligned}
$$

где индексы у знака Smbl указывают, относительно каких упорядоченных переменных берется символ (см., например, [22]).

ДокАЗАТЕЛЬСТво. Пусть $\psi(p, t)$ - подынтегральное выражение в (2.4). Имеем (см. [22])

$\psi(p, t)=G\left(p-i \frac{\partial}{\partial t}\right)\left(F\left(p-i \frac{\partial}{\partial t}\right)-F(p)\right) f(t)=-i G\left(p-i \frac{\partial}{\partial t}\right) \Phi\left(p,-i \frac{\partial}{\partial t}\right) f^{\prime}(t)$,

где

$$
\Phi(p, \tau)=\int_{0}^{1} F^{\prime}(p+\lambda \tau) d \tau
$$

в силу леммы Адамара. Пусть

$$
P(p, \xi)=-i G(p+\xi) \Phi(p, \xi) .
$$


Мы имеем

$$
\begin{aligned}
H(p) & =\int_{-\infty}^{\infty} P\left(p,-i \frac{\partial}{\partial t}\right) f^{\prime}(t) d t \\
& =\left\langle 1, P\left(p,-i \frac{\partial}{\partial t}\right) f^{\prime}(t)\right\rangle=\left\langle P\left(p, i \frac{\partial}{\partial t}\right) 1, f^{\prime}(t)\right\rangle \\
& =\left\langle P(p, 0) 1, f^{\prime}(t)\right\rangle=P(p, 0)\left\langle 1, f^{\prime}(t)\right\rangle=P(p, 0)(f(+\infty)-f(-\infty)) .
\end{aligned}
$$

Здесь угловые скобки означают спаривания между пространствами медленно растуших распределений быстроубывающих функций. Лемма доказана.

Применяя эту лемму и стандартную формулу следа, получаем

$$
\operatorname{ind}\left(T_{\gamma}\right)=\frac{1}{2 \pi i} \int_{\mathscr{L}_{\gamma}} \operatorname{trace}\left(R(p)-R_{1}(p)\right) \frac{\partial T}{\partial p}(p) d p+\operatorname{trace}_{\gamma} \widetilde{Q}
$$

где $\mathscr{L}_{\gamma}=\{\operatorname{Im} p=\gamma\}$ - весовая прямая.

Поскольку символ $\widetilde{Q}$ голоморфен, отсюда следует, что trace $\gamma \widetilde{Q}$ на самом деле не зависит от $\gamma$, и мы получаем следуюшую теорему

ТЕОРема 2.1 ("относительная формула индекса"). Пусть $\gamma_{1} \leqslant \gamma_{2}$ и конормальный символ обратим на весовых прямых $\mathscr{L}_{\gamma_{1}}$ и $\mathscr{L}_{\gamma_{2}}$. Тогда

$$
\operatorname{ind}\left(T_{\gamma_{2}}\right)-\operatorname{ind}\left(T_{\gamma_{1}}\right)=\sum_{\gamma_{1}<\operatorname{Im} p_{j}<\gamma_{2}} \operatorname{trace}\left(\operatorname{Res}_{p_{j}} T^{-1}(p) \frac{\partial T(p)}{\partial p}\right),
$$

где $p_{j}$ - полюсы семейства $T^{-1}(p)=R(p)$.

ДокАЗАТЕЛЬСтво. Достаточно применить теорему Коши о вычетах и учесть тот факт, что член $R_{1}(p) \partial T(p) / \partial p$ голоморфен и поэтому не дает вклад в вычеты полюсов в подынтегральной функции.

2.2. Условие симметрии и склейка. Предположим, что конормальный символ $T(p)$ оператора $T=T(g, a)$ удовлетворяет следующему условию симметрии:

$$
T(-p)=T(p) .
$$

Случай, когда симметрия имеет место не относительно начала координат, а относительно некоторой точки $p=p_{0}$, может быть редуцирован к данному преобразованием подобия с оператором $U=e^{-p_{0} t}$. Условие симметрии [19]

$$
\sigma_{1} T\left(p_{0}-p\right)=T\left(p_{0}+p\right) \sigma_{2},
$$

включающее гомоморфизмы расслоений $\sigma_{1}$ и $\sigma_{2}$, а также некоторые и более обшие условия симметрии, может быть также включено в нашу схему без каких-либо дополнительных идей. Для того чтобы не утяжелять изложения, мы ограничимся здесь простейшим условием (2.7).

Приведем пример ИО $~$ с конормальным символом, удовлетворяюшим условию симметрии (2.7). Этот ИОФ соответствует некоторому каноническому преобразованию $g$. Опишем $g$ лишь вблизи конической точки (что только и является существенным для конормального символа). Будем работать в цилиндрических координатах на $T^{*} K$, т. е. в координатах $(t, \omega, p, q),(\omega, q) \in T^{*} \Omega, t \in \mathbb{R}, p \in \mathbb{R},-$ 
конормальная переменная (двойственная к $t$ ). Будем описьвать каноническое преобразование

$$
\begin{gathered}
g: T_{0}^{*} M \rightarrow T_{0}^{*} M, \\
(t, \omega, p, q) \mapsto\left(t^{\prime}, \omega^{\prime}, p^{\prime}, q^{\prime}\right)
\end{gathered}
$$

с помощью производящей функции. В простейшем случае производящая функция $S$ зависит от переменных $\left(p, q, t^{\prime}, \omega^{\prime}\right)$ (неособая карта в определении канонического оператора) и задает каноническое преобразование по формулам

$$
\begin{array}{ll}
t=t^{\prime}+\frac{\partial S}{\partial p}\left(p, q, t^{\prime}, \omega^{\prime}\right), & \omega=\omega^{\prime}+\frac{\partial S}{\partial q}\left(p, q, t^{\prime}, \omega^{\prime}\right), \\
p^{\prime}=p+\frac{\partial S}{\partial t^{\prime}}\left(p, q, t^{\prime}, \omega^{\prime}\right), & q^{\prime}=q^{\prime}+\frac{\partial S}{\partial \omega^{\prime}}\left(p, q, t^{\prime}, \omega^{\prime}\right) .
\end{array}
$$

Конормальный символ имеет вид

$$
T_{0}(p)=\exp \left\{i S\left(p, i \frac{\partial}{\partial \omega},+\infty, \stackrel{2}{\omega}\right)\right\} a\left(p, i \frac{\partial}{\partial \omega},+\infty, \stackrel{2}{\omega}\right)
$$

и можно добиться выполнения условия симметрии, выбирая амплитуду $a$ подходяшим образом, при условии, что

$$
S(p, q,+\infty, \omega) \equiv S(-p, q,+\infty, \omega)
$$

Простейший конкретньй пример строится следующим образом. Пусть $\Omega=S^{1}$ окружность с угловой координатой $\omega$. Рассмотрим (глобальную) производящую функцию вида

$$
S \equiv S(p, q)=\sqrt{p^{2}+q^{2}}
$$

Однородное каноническое преобразование задается формулами

$$
p^{\prime}=p, \quad q^{\prime}=q, \quad t^{\prime}=t-\frac{p}{\sqrt{p^{2}+q^{2}}}, \quad \omega=\omega-\frac{q}{\sqrt{p^{2}+q^{2}}}
$$

Для перехода к соответствуюшему ИОФ аналитически сгладим функцию $S(p, q)$, свернув ее с преобразованием Фурье четной гладкой функции с компактным носителем. Тогда сглаженная функция вновь удовлетворяет условию $S(-p, q)=$ $S(p, q)$, и, выбирая единичную амплитуду, мы получаем симметричный конормальный символ:

$$
T_{0}(p) \phi(\omega)=\sum_{k=-\infty}^{\infty} \exp (i(S(p, k)+k \omega)) \phi_{k},
$$

где $\phi_{k}-$ коэффициенты Фурье функции $\phi$.

Предположим, что конормальный символ $T(p)$ обратим на весовой линии $\mathscr{L}_{\gamma}$. Тогда в силу $(2.7)$ он также обратим на линии $\mathscr{L}_{-\gamma}$. Вычислим сумму ind $\left(T_{\gamma}\right)+$ 


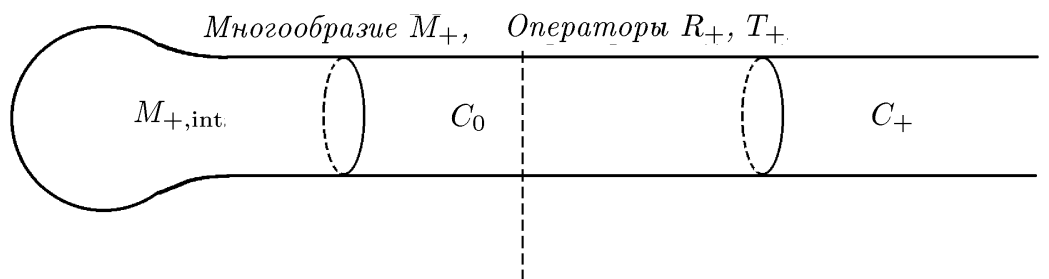

Многообразие $M_{-}$, Операторы $R_{-}, T_{-}$

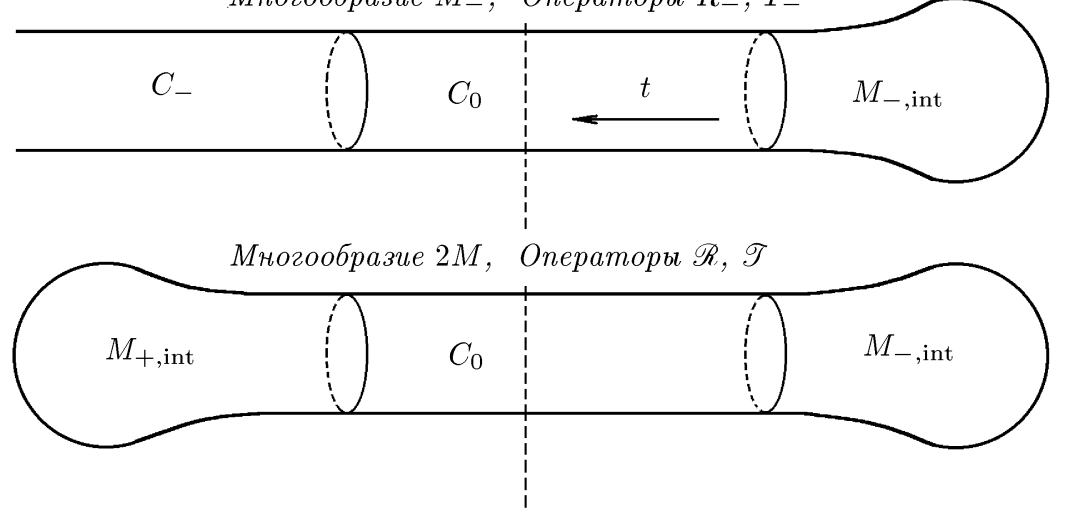

Многообразие $C$, Oператоры $R_{0}, T_{0}$

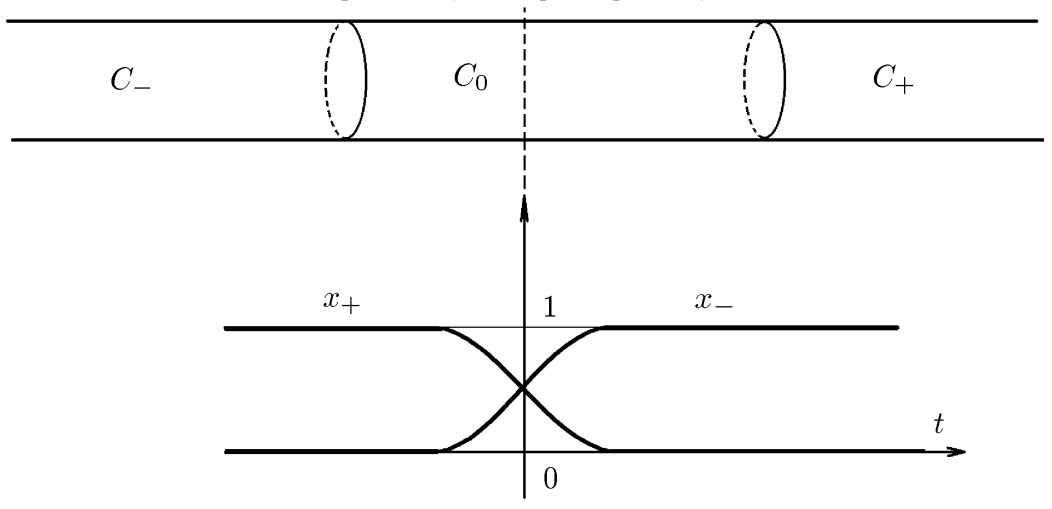

Рис. 1. Два экземпляра многообразия $M$, дубль $2 M$ и цилиндр $C$

ind $\left(T_{-\gamma}\right)$ и покажем, что она определяется только каноническим преобразованием $g$ и главным символом $a$. Для того чтобы это сделать, нам необходимы некоторые геометрические и аналитические конструкции.

а) Геометрические объекты (см. рис. 1).

Рассмотрим два экземпляра $M_{+}$и $M_{-}$многообразия $M$. Мы будем изображать их как многообразия с цилиндрическими концами (см. п. 1.1). Это означает, что в окрестности особой точки вместо конических используются цилиндрические координаты, так что соответствующая часть многообразия выглядит, как "уходящий в бесконечность" цилиндр $\Omega \times(0,+\infty)$. На первом экземпляре $M_{+}$цилиндрический конец есть $C_{0} \cup C_{+}$, и внутренняя часть многообразия обозначается через $M_{+}$,int . Далее, разделим цилиндрический конец на две части $C_{0}$ и $C_{+}$, где $C_{0}$ есть достаточно длинный (ниже увидим, какой именно длины) конечный цилиндр с базой $\Omega$, 
а $C_{+}$- оставшаяся бесконечная часть. Удобно выбрать цилиндрическую координату $t$ (определенную на цилиндрическом конще) так, чтобы $\{t=0\}$ было бы в точности среднее сечение цилиндра $C_{0}$.

Для второго экземпляра $M_{-}$обозначим внутреннюю часть через $M_{- \text {,int }}$ (она изоморфна $\left.M_{+, \text {int }}\right)$ и цилиндрический конец через $C_{0} \cup C_{-}\left(C_{-}\right.$изоморфно $\left.C_{+}\right)$. Заметим, что на цилиндрических частях всех многообразий, показанных на рис. 1 , за исключением $M_{-}$, переменная $t$ увеличивается слева направо; для $M_{-}$направление $t$-оси противоположно.

Теперь выбросим $C_{+}$из $M_{+}$и $C_{-}$из $M_{-}$и склеим полученное многообразие вдоль $C_{0}$, отождествляя каждое сечение $\{t\} \times \Omega \subset C_{0} \subset M_{+}$с соответствующим сечением $\{-t\} \times \Omega \subset C_{0} \subset M_{-}$. В результате получим многообразие, обозначаемое через $2 M$ (см. рис. 1). Это многообразие будем называть дублем многообразия $M$. Склейка многообразий поднимается до соответствующей склейки соответствующих кокасательных расслоений (значения конормальной переменной $p$ с одним и тем же модулем и противоположными знаками на $T^{*} M_{+}$и $T^{*} M_{-}$отождествляется). Легко видеть, что функция $F$ в $(2.1)$ является нечетной функцией переменной $p$ при условиях симметрии (2.7), так что преобразование $g$ "пропускается через склейку", и мы получаем каноническое преобразование

$$
2 g: T_{0}^{*}(2 M) \rightarrow T_{0}^{*}(2 M)
$$

Наконец, введем бесконечный цилиндр $C$ с базой $\Omega$, который также представляется как объединение:

$$
C=C_{-} \cup C_{0} \cup C_{+}
$$

б) Аналитические объекты.

Напомним, что $R(p)=(T(p))^{-1}$. Оператор $R(p)$ не имеет полюсов на линии $\mathscr{L}_{\gamma}$, и операторы

$$
T_{0}=T\left(-i \frac{\partial}{\partial t}\right), \quad R_{0}=R\left(-i \frac{\partial}{\partial t}\right)
$$

корректно определены в шкале $\left\{H_{\gamma}^{s}(C)\right\}$ и удовлетворяют соотношениям

$$
T_{0} R_{0}=1, \quad R_{0} T_{0}=1
$$

Пусть $\chi_{+}(t), \chi_{-}(t)$ - разбиение единищы на вещественной прямой (см. рис. 1$)$. Предположим, что эти функции постоянны вне достаточно малой окрестности $t=0$; тогда они могут быть естественным образом продолжены до разбиения единищы на всех четырех многообразиях, показанных на рисунке. Будем во всех случаях использовать те же самые обозначения $\chi_{+}(t), \chi_{-}(t)$.

Пусть $T_{+}$- оператор $T$ в $H_{\gamma}^{s}\left(M_{+}\right)$и $T_{-}$- оператор $T$ в $H_{-\gamma}^{s}\left(M_{-}\right)$, и аналогично для $R_{+}$и $R_{-}$, где $R$ есть регуляризатор оператора $T$ (см. п. 2.1). Не уменьшая обшности, можно предположить, что справедливы следующие условия (возможно, после добавления компактных сглаживающих операторов к операторам $T_{ \pm}$и $R_{ \pm}$, которые не меняют индекс).

1. Для достаточно больших $t, \tau$ (т.е. в $C_{+}$) ядра операторов $T_{+}$и $R_{+}$совпадают с ядрами операторов $T_{0}$ и $R_{0}$ соответственно. Полный операторнозначный символ оператора $T_{+}$(соответственно $R_{+}$) относительно квантования $\stackrel{1}{\partial} / \partial t, \stackrel{2}{t}$ совпадает с символом оператора $T_{-}\left(\right.$соответственно $\left.R_{-}\right)$в $C_{0} \cup C_{+}$. Аналогичные 
условия справедливы для $T_{-}$и $R_{-}$. (Заметим, что условия, налагаемые на "+" и "-"-операторы, совместимы с условием (2.7).)

2. Пусть $K(x, y)$ - ядро любого из операторов $T_{+}$и $R_{+}$. Если хотя бы одна из точек $x$ и $y$ не принадлежит $C_{+}$, то $K(x, y)=0$ вне достаточно малой окрестности проекции $M_{+} \times M_{+}$графика соответствующего каноническго преобразования (т.е. $g$ или $g^{-1}$ ).

3. В частности, пересечение supp $K_{T_{ \pm}} \cap\left(C_{0} \times C_{0}\right)$ лежит в полосе $\left\{|t-\tau|<t_{0}\right\}$, где $t_{0}$ мало в сравнении с длиной $C_{0}$ (скажем, меньше, чем $1 / 10$ этой длины; достаточно взять $t_{0}>\sup |F(\omega, p, q)|$ (см. $\left.(2.1)\right)$ и затем взять $C_{0}$ длиннее, чем $\left.10 t_{0}\right)$.

Окончательно, пусть $\mathscr{T}$ и $\mathscr{R}$ - интегральные операторы Фурье в шкале Соболева на многообразии $2 M$, ассоциированные с каноническими преобразованиями $2 g$ и $(2 g)^{-1}$ соответственно, которые совпадают по модулю сглаживающих операторов с операторами $T_{+}$и $R_{+}$в $M_{+, \text {int }} \cup C_{0}$ и с операторами $T_{-}$и $R_{-}$в $M_{- \text {,int }} \cup C_{0}$. Предположим также, что носители ядер Шварца операторов $\mathscr{T}$ и $\mathscr{R}$ лежат в малых окрестностях проекции графиков соответствующих канонических преобразований. (Это техническое требование может быть всегда удовлетворено добавлением подходящих сглаживаюших операторов, которые не влияют на индекс оператоpa $\mathscr{T}$.)

Теперь мы установим следующий замечательный результат, касающийся соотношения между индексами операторов $T_{ \pm}$и индексом "склеенного" оператора $\mathscr{T}$ на $2 M$.

Теорема 2.2. Справедливо следующее равенство:

$$
\operatorname{ind} T_{+}+\operatorname{ind} T_{-} \equiv \operatorname{ind}\left(T_{\gamma}\right)+\operatorname{ind}\left(T_{-\gamma}\right)=\text { ind } \mathscr{T} \text {. }
$$

ДокАЗАТЕЛЬСТво. Введем для краткости следующее обозначение. Будем писать

$$
\operatorname{trace} A-\operatorname{trace} B=\left[\begin{array}{l}
A \\
B
\end{array}\right]_{S},
$$

где $A$ и $B$ - операторы на многообразии $S$. (В качестве $S$ всегда будет использоваться одно из многообразий, показанных на рис. 1 , а пространства, в которых берутся следы, суть $L^{2}$-пространства в соответствующих соболевских шкалах, в которых эти операторы действуют.) Обозначение

$$
\llbracket \begin{aligned}
& A \\
& B
\end{aligned} \rrbracket_{S}
$$

означает в точности то же, что и $\left[\begin{array}{l}A \\ B\end{array}\right]_{S}$, но будет использовано для указания, что

$$
\left[\begin{array}{l}
A \\
B
\end{array}\right]_{S}=0
$$

В частности, мы будем всегда писать $\left.\llbracket \begin{array}{l}A B \\ B A\end{array}\right]_{S}$, если операторы $A$ и $B$ на $S$ удовлетворяют предположениям леммы 2.1. 
Основное средство в доказательстве этой теоремы - это переход от одного многообразия к другому при вычислении следов. Это возможно всегда, когда операторы могут быть проинтерпретированы либо на одном многообразии, либо на другом. Например, в силу наших условий на носители ядер Шварца, ядра Шварца операторов $\chi_{+} R_{+} T_{+}$и $T_{+} \chi_{+} R_{+}$имеют носители во множестве $\left\{M_{+}\right.$,int $\left.\cup C_{0}\right\} \times$ $\left\{M_{+}\right.$,int $\left.\cup C_{0}\right\}$, и поэтому каждый из этих операторов может быть рассмотрен на многообразии $M_{+}$или $2 M$, в зависимости от того, как это требуется. Впоследствии эти очевидные, но длинные объяснения об обосновании перехода от одного многообразия к другому будут опускаться.

Итак, вычислим ind $\mathscr{T}$ (все циклические преобразования множителей справедливы в силу леммы 2.1).

Имеем

$$
\text { ind } \mathscr{T}=\operatorname{trace}(1-\mathscr{R} \mathscr{T})-\operatorname{trace}(1-\mathscr{T} \mathscr{R})=\left[\begin{array}{c}
1_{2 M}-\mathscr{R} \mathscr{T} \\
1_{2 M}-\mathscr{T} \mathscr{R}
\end{array}\right]_{2 M}
$$

(здесь $1_{2 M}$ - тождественный оператор на многообразии $2 M$ ). Впоследствии для краткости будем опускать нижний индекс, обозначающий многообразие, если это многообразие есть $2 M$.

Сделаем некоторые преобразования. Имеем

$$
\begin{aligned}
{\left[\begin{array}{c}
1-\mathscr{R} \mathscr{T} \\
1-\mathscr{T} \mathscr{R}
\end{array}\right]=} & {\left[\begin{array}{l}
1-\chi_{+} \mathscr{R} \mathscr{T}-\chi_{-} \mathscr{R} \mathscr{T} \\
1-\mathscr{T} \chi_{+} \mathscr{R}-\mathscr{T} \chi_{-} \mathscr{R}
\end{array}\right] } \\
= & {\left[\begin{array}{l}
1-\chi_{+} R_{+} \mathscr{T}-\chi_{-} R_{-} \mathscr{T} \\
1-\mathscr{T} \chi_{+} R_{+}-\mathscr{T} \chi_{-} R_{-}
\end{array}\right] } \\
& +\llbracket\left[\begin{array}{c}
\chi_{+}\left(R_{+}-\mathscr{R}\right) \mathscr{T} \\
\mathscr{T} \chi_{+}\left(R_{+}-\mathscr{R}\right)
\end{array}\right]+\llbracket\left[\begin{array}{c}
\chi_{-}\left(R_{-}-\mathscr{R}\right) \mathscr{T} \\
\mathscr{T} \chi_{-}\left(R_{-}-\mathscr{R}\right)
\end{array}\right] \\
= & {\left[\begin{array}{l}
1-\chi_{+} R_{+} T_{+}-\chi_{-} R_{-} T_{-} \\
1-T_{+} \chi_{+} R_{+}-T_{-} \chi_{-} R_{-}
\end{array}\right] } \\
& +\llbracket\left[\begin{array}{l}
\chi_{+} R_{+}\left(T_{+}-\mathscr{T}\right) \\
\left(T_{+}-\mathscr{T}\right) \chi_{+} R_{+}
\end{array}\right]+\llbracket\left[\begin{array}{l}
\chi_{-} R_{-}\left(T_{-}-\mathscr{T}\right) \\
\left(T_{-}-\mathscr{T}\right) \chi_{-} R_{-}
\end{array}\right] .
\end{aligned}
$$

Пусть теперь $e-$ функция с носителем в $M_{+, \text {int }} \cup C_{0}$ такая, что

$$
e T_{+} \chi_{+} R_{+}=T_{+} R_{+} \chi_{+}, \quad e \chi_{+}=\chi_{+} .
$$

Положим

$$
A=e-e T_{-} \chi_{-} R_{-}, \quad B=1-A .
$$

Тогда оператор $A$ может рассматриваться как оператор на $M_{+}$, оператор $B$ - как оператор на $M_{-}$и, более того, операторы

$$
A-T_{+} \chi_{+} R_{+}=e\left(1-T_{+} \chi_{+} R_{+}-T_{-} \chi_{-} R_{-}\right), \quad B-T_{-} \chi_{-} R_{-}
$$

принадлежат trace-классу. Поэтому мы можем записать

$$
\begin{aligned}
& {\left[\begin{array}{c}
1-\chi_{+} R_{+} T_{+}-\chi_{-} R_{-} T_{-} \\
1-T_{+} \chi_{+} R_{+}-T_{-} \chi_{-} R_{-}
\end{array}\right]} \\
& \quad=\left[\begin{array}{c}
\chi_{+}-\chi_{+} R_{+} T_{+} \\
A-T_{+} \chi_{+} R_{+}
\end{array}\right]_{M_{+}}+\left[\begin{array}{c}
\chi_{-}-\chi_{-} R_{-} T_{-} \\
B-T_{-} \chi_{-} R_{-}
\end{array}\right]_{M_{-}}
\end{aligned}
$$


Преобразуем первый член в правой части. Имеем (ниже для краткости пишем $1_{+}$вместо $\left.1_{M_{+}}\right)$

$$
\begin{aligned}
& {\left[\begin{array}{c}
\chi_{+}-\chi_{+} R_{+} T_{+} \\
A-T_{+} \chi_{+} R_{+}
\end{array}\right]_{M_{+}}=\left[\begin{array}{c}
1_{+}-R_{+} T_{+} \\
1_{+}-T_{+} R_{+}
\end{array}\right]_{M_{+}}} \\
& \quad-\left[\begin{array}{c}
\chi_{-}-\chi_{-} R_{+} T_{+} \\
1_{+}-A-T_{+} \chi_{-} R_{+}
\end{array}\right]_{M_{+}}=\operatorname{Ind} T_{+}-\left[\begin{array}{c}
\chi--\chi_{-} R_{+} T_{+} \\
1_{+}-A-T_{+} \chi_{-} R_{+}
\end{array}\right]_{M_{+}} .
\end{aligned}
$$

Отсюда очевидно, что оператор $1_{+}-A$ может быть представлен как оператор на многообразии $C$; поэтому

$$
\begin{aligned}
{\left[\begin{array}{c}
\chi_{-}-\chi_{-} R_{+} T_{+} \\
1_{+}-A-T_{+} \chi_{-} R_{+}
\end{array}\right]_{M_{+}} } & =\left[\begin{array}{c}
\chi-\chi \chi_{-} R_{+} T_{+} \\
\left(1_{+}-A\right)-T_{+} \chi_{-} R_{+}
\end{array}\right]_{C} \\
& =\left[\begin{array}{c}
\chi_{-}-\chi_{-} R_{+} T_{0} \\
\left(1_{+}-A\right)-T_{0} \chi_{-} R_{+}
\end{array}\right]_{C}+\llbracket\left[\begin{array}{l}
\chi_{-} R_{+}\left(T_{0}-T_{+}\right) \\
\left(T_{0}-T_{+}\right) \chi_{-} R_{+}
\end{array}\right]_{C} \\
& =\left[\begin{array}{c}
\chi_{-}-\chi_{-} R_{0} T_{0} \\
\left(1_{+}-A\right)-T_{0} \chi_{-} R_{0}
\end{array}\right]_{C}+\llbracket\left[\begin{array}{c}
\chi_{-}\left(R_{0}-R_{+}\right) T_{0} \\
T_{0} \chi_{-}\left(R_{0}-R_{+}\right)
\end{array} \|_{C}\right. \\
& =\left[\begin{array}{c}
0 \\
\left(1_{+}-A\right)-T_{0} \chi_{-} R_{0}
\end{array}\right]_{C}
\end{aligned}
$$

(напомним, что $R_{0} T_{0}=1_{C}$ ).

Аналогично

$$
\left[\begin{array}{c}
\chi_{-}-\chi_{-} R_{-} T_{-} \\
B-T_{-} \chi_{-} R_{-}
\end{array}\right]_{M_{-}}=\operatorname{Ind} T_{-}+\left[\begin{array}{c}
0 \\
\left(1_{-}-B\right)-T_{0} \chi_{+} R_{0}
\end{array}\right]_{C}
$$

Остается доказать, что сумма остаточных членов в (2.16) и (2.17) равно нулю. Имеем

Далее,

$$
\begin{aligned}
& {\left[\begin{array}{c}
0 \\
\left(1_{+}-A\right)-T_{0} \chi_{-} R_{0}
\end{array}\right]_{C}+\left[\begin{array}{c}
0 \\
\left(1_{-}-B\right)-T_{0} \chi_{+} R_{0}
\end{array}\right]_{C}} \\
& \quad=\left[\begin{array}{c}
0 \\
\left(1_{+}-A\right)+\left(1_{-}-B\right)-1_{C}
\end{array}\right]_{C} .
\end{aligned}
$$

и соответственно

$$
1_{C}=\chi_{+}+\chi_{-}
$$

$$
\left(1_{+}-A\right)+\left(1_{-}-B\right)-1_{C}=P_{1}+P_{2}
$$

где

$$
P_{1}=\left(1_{+}-A\right)-\chi_{+}, \quad P_{2}=\left(1_{-}-B\right)-\chi_{-} .
$$

Ядра Шварца операторов $P_{1}$ и $P_{2}$ имеют носители в $C_{0} \times C_{0}$ и достаточно показать, что сумма этих ядер равна здесь нулю. Будем писать $D_{1} \sim D_{2}$, если ядра Шварца $D_{1}$ и $D_{2}$ совпадают в $C_{0} \times C_{0}$. (Многообразия, на которых операторы $D_{1}$ и $D_{2}$ определены, не обязаны совпадать, но, разумеется, оба многообразия должны содержать $C_{0}$.) Имеем

$$
P_{1} \sim \chi_{-}-A, \quad P_{2} \sim \chi_{+}-B,
$$

где правые части понимаются как операторы на дубле $2 M$. Но

$$
\chi_{-}-A+\chi_{+}-B=1_{2 M}-1_{2 M}=0 .
$$

Этим завершается доказательство теоремы 2.2 . 
2.3. Теорема об индексе. Теперь можно сформулировать и доказать основной результат об индексе ИОФ. Пусть $T=T(g, a)-$ ИО $\Phi$, удовлетворяющий условию симметрии (2.7), $\gamma \geqslant 0$ и весовая прямая $\mathscr{L}_{\gamma}$ не содержит полюсов конормального символа $T(p)$. (Для $\gamma<0$ утверждение совершенно аналогично.)

ТЕОРема 2.3. При указанных условиях

$$
\operatorname{ind}\left(T_{\gamma}\right)=\frac{1}{2} \text { ind } \mathscr{T}-\frac{1}{2} \sum_{-\gamma<\operatorname{Im} p_{j}<\gamma} \operatorname{Res}_{p_{j}}\left(T^{-1}(p) \frac{\partial T(p)}{\partial p}\right)
$$

где $\mathscr{T ~ - ~ И О Ф ~ н а ~ д у б л е ~} 2 M$, построенный в доказательстве теоремь 2.2, а $p_{j}-$ полюсы функиии $T^{-1}(p)$.

Доказательство немедленно вытекает из теорем 2.1 и 2.2.

СледСТВиЕ 2.1 (индекс квантованных канонических преобразований). Пусть $T=T(g)$ - квантованное каноническое преобразование и $T(p)$ - его конормальный символ, $\gamma \geqslant 0$. Предположим, ито весовая линия $\mathscr{L}_{\gamma}$ не содержит полюсов функиии $T(p)$. Тогда если выполнено условие симметрии (2.7), то справедлива формула

$$
\operatorname{ind}\left(T_{\gamma}\right)=\frac{1}{2} c-\operatorname{deg}(\widetilde{2 g})-\frac{1}{2} \sum_{-\gamma<\operatorname{Im} p_{j}<\gamma} \operatorname{Res}_{p_{j}}\left(T^{-1}(p) \frac{\partial T(p)}{\partial p}\right)
$$

дде с-deg - контактная степень [6], $\widetilde{2 g}$ - контактное преобразование, соответствующее $2 g$, и $p_{j}$ - полюсы функции $T^{-1}(p)$.

ЗАмЕчАниЕ 2.1. Для случая условия симметрии (2.8) формула индекса получает (в предположении, что $\gamma \geqslant \operatorname{Im} p_{0}$ ) следующий вид:

$$
\operatorname{ind}\left(T_{\gamma}\right)=\frac{1}{2} c-\operatorname{deg}(2 g)-\frac{1}{2} \sum_{2 \operatorname{Im} p_{0}-\gamma<\operatorname{Im} p_{j}<\gamma} \operatorname{Res}_{p_{j}}\left(T^{-1}(p) \frac{\partial T(p)}{\partial p}\right)
$$

ДокАЗАТЕЛЬСтво слЕДствия 2.1. Оператор $\mathscr{T}$ является квантованным контактным преобразованием над $2 M$, соответствуюшим (однородному) каноническому преобразованию $2 g$. Как показано в [6], такое преобразование унитарно эквивалентно соответствующему квантованному контактному преобразованию. Таким образом, можно использовать формулу индекса, полученную в [6] для квантованных контактных преобразований над конфигурационным пространством, которое является гладким замкнутым многообразием. Именно, мы имеем

$$
\text { ind } \mathscr{T}=c-\operatorname{deg}(\widetilde{2 g})
$$

Остается применить теорему 2.3. 


\section{§3. Приложение}

ДОКАЗАТЕЛЬСТвО ЛЕММЫ 1.3. Мы имеем

$$
T=\sum T_{j}
$$

где сумма конечна и операторы $T_{j}$ соответствуют локальным каноническим операторам в канонических картах. Таким образом, необходимо доказать, что $T_{j}^{*} T_{k}$ является псевдодифференциальным оператором. Если операторы $T_{j}$ и $T_{k}$ соответствуют локальным каноническим операторам в картах, чьи проекции на многообразие $M$ не содержат конических точек, нужное утверждение может быть доказано стандартным образом (см. [14], [16]). Таким образом, необходимо изучить лишь случай, когда, по крайней мере, один из этих операторов соответствует канонической карте в окрестности конической точки. Для простоты изложения рассмотрим только случай $j=k$, и предположим, что каноническая карта неособая: $\bar{I}=\varnothing$ и носитель элемента $\pi_{1}^{*} a$ содержится в этой карте. Тогда

$$
[T(g, a) u](t, \omega)=\left(\frac{i}{2 \pi}\right)^{n} \int e^{i\left(p t+S_{1}\left(e^{-t}, \omega, p, q\right)\right)} b\left(e^{-t}, \omega, p, q\right) \widetilde{u}(p, q) d p d q,
$$

где $p t+S_{1}\left(e^{-t}, \omega, p, q\right)$ - производящая функция преобразования $g$ в данной канонической карте, $b=\left(\pi_{1}^{*} a\right) \sqrt{\mu_{1, \ldots, n}} \chi(p, q), \chi(p, q)$ - срезающая функция, равная 1 в окрестности бесконечности, $\mu_{1}, \ldots, n-$ плотность меры $\mu$ в координатах $(\tau, \psi, p, q)$ канонической карты, $n=\operatorname{dim} M$ и

$$
\widetilde{u}(p, q)=\left(\frac{1}{2 \pi i}\right)^{n} \int e^{-i(p t+\omega q)} u(t, \omega) d t d \omega
$$

- преобразование Фурье функции $u(t, \omega)$. Уравнение (3.1) может быть переписано в виде

$$
T(g, a)=\exp \left\{i \Phi\left(e^{-t}, \stackrel{2}{\omega}, \hat{p}, \hat{q}\right)\right\} b\left(e^{2}-t, \stackrel{2}{\omega}, \hat{p}, \hat{p}, \hat{q}\right)
$$

где

$$
\begin{gathered}
\Phi\left(e^{-t}, \omega, p, q\right)=S_{1}\left(e^{-t}, \omega, p, q\right)-p q, \\
\hat{p}=-i \frac{\partial}{\partial t}, \quad \hat{q}=-i \frac{\partial}{\partial \omega} .
\end{gathered}
$$

Для доказательства утверждения этой леммы мы используем метод, предложенньй в [23]. Имеем

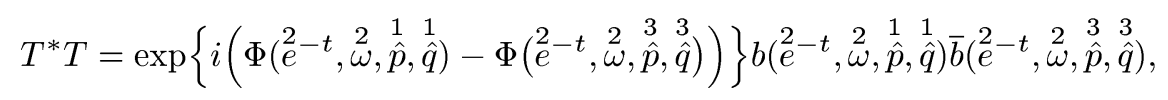

где черта над $b$ означает комплексное сопряжение. Для краткости обозначим $(t, \omega)=x,(p, q)=\xi$. Тогда

$$
T^{*} T=\exp \{i[S(2 \stackrel{2}{x}, \hat{\xi})-S(\stackrel{2}{x}, \stackrel{3}{\hat{\xi}})]\} \varphi\left(\stackrel{2}{x}, \frac{1}{\hat{\xi}}\right) \bar{\varphi}(\stackrel{2}{x}, \hat{\xi}),
$$


где

$$
S(x, \xi)=\Phi\left(e^{-t}, \omega, p, q\right), \quad \varphi(x, \xi)=b\left(e^{-t}, \omega, p, q\right) .
$$

Применим теперь оператор $T^{*} T$ к произвольной функции $f(x)$. Имеем

$$
\begin{aligned}
T^{*} T f= & \left(\frac{1}{2 \pi}\right)^{2 n} \int e^{i\left(\xi x-\xi^{\prime} x^{\prime \prime}\right)} f\left(x^{\prime \prime}\right)\left\{\int \exp \left\{i\left[\left(\xi^{\prime}-\xi\right) x^{\prime}+S\left(x^{\prime}, \xi^{\prime}\right)-S\left(x^{\prime}, \xi\right)\right]\right\}\right. \\
& \left.\times \varphi\left(x^{\prime}, \xi^{\prime}\right) \bar{\varphi}\left(x^{\prime}, \xi\right) d x^{\prime}\right\} d x^{\prime \prime} d \xi d \xi^{\prime}
\end{aligned}
$$

Поскольку функция

$$
S_{1, \ldots, n}(x, \xi)=x \xi+S(x, \xi)
$$

является производящей функщией преобразования $g$ в рассматриваемой канонической карте, то якобиан

$$
J_{0}(x, \xi)=\operatorname{det}\left(E+\frac{\partial^{2} S}{\partial x \partial \xi}(x, \xi)\right)
$$

не обращается в нуль в канонической карте (и, в частности, на носителе $\varphi(x, \xi)$ ), и, более того, уравнение

$$
\xi+\frac{\partial S}{\partial x}(x, \xi)=\eta
$$

имеет не более одного решения на носителе функции $\varphi(x, \xi)$ для каждого $\eta \in \mathbb{R}^{n}$. Этот факт может быть переформулирован в виде следующего неравенства:

$$
\xi+\frac{\partial S}{\partial x}(x, \xi) \neq \xi^{\prime}+\frac{\partial S}{\partial x}\left(x, \xi^{\prime}\right)
$$

справедливого для $(x, \xi) \in \operatorname{supp} \varphi,\left(x, \xi^{\prime}\right) \in \operatorname{supp} \varphi, \xi \neq \xi^{\prime}$.

Уравнение (3.6) показывает, что можно интегрировать по частям относительно $x^{\prime}$ во внутреннем интеграле (3.4) для $\xi \neq \xi^{\prime}$. Для того чтобы использовать этот факт, введем гладкое разбиение единицы:

$$
1=\chi_{1}\left(\xi, \xi^{\prime}\right)+\chi_{2}\left(\xi, \xi^{\prime}\right)+\chi_{3}\left(\xi, \xi^{\prime}\right)
$$

где $\chi_{i}, i=1,2,3,-$ однородные функции нулевого порядка для достаточно больших значений $|\xi|+\left|\xi^{\prime}\right|$,

$$
\begin{array}{ll}
\chi_{1}=0, & \frac{\left|\xi-\xi^{\prime}\right|}{|\xi|} \geqslant 2 \varepsilon \\
\chi_{2}=0, & \frac{\left|\xi-\xi^{\prime}\right|}{|\xi|} \leqslant \varepsilon \\
\chi_{3}=0, & |\xi|+\left|\xi^{\prime}\right| \geqslant 2 \\
\chi_{3}=1, & |\xi|+\left|\xi^{\prime}\right| \leqslant 1
\end{array}
$$

(здесь $\varepsilon>0$ - достаточно малое число, которое будет выбрано позднее). Теперь перепишем уравнение (3.4) в виде

$$
T^{*} T f=\sum_{j=1}^{3} P_{j} f,
$$


где

$$
\begin{aligned}
P_{j} f= & \left(\frac{1}{2 \pi}\right)^{2 n} \int e^{i\left(\xi x-\xi^{\prime} x^{\prime \prime}\right)} f\left(x^{\prime \prime}\right)\left\{\int \exp \left\{i\left[\left(\xi^{\prime}-\xi\right) x^{\prime}+S\left(x^{\prime}, \xi^{\prime}\right)-S\left(x^{\prime}, \xi\right)\right]\right\}\right. \\
& \left.\times \varphi\left(x^{\prime}, \xi^{\prime}\right) \bar{\varphi}\left(x^{\prime}, \xi\right) \chi_{j}\left(\xi, \xi^{\prime}\right) d x^{\prime}\right\} d x^{\prime \prime} d \xi d \xi^{\prime}
\end{aligned}
$$

Очевидно, что

$$
P_{3}=P_{3}(\stackrel{1}{\hat{\xi}}, \stackrel{2}{x}, \stackrel{3}{\hat{\xi}})
$$

где

$$
P_{3}\left(\xi^{\prime}, x, \xi\right)=\chi_{3}\left(\xi, \xi^{\prime}\right) e^{i\left[S\left(x, \xi^{\prime}\right)-S(x, \xi)\right]} \varphi\left(x, \xi^{\prime}\right) \bar{\varphi}(x, \xi)
$$

- гладкая функция, финитная по переменным $\left(\xi, \xi^{\prime}\right)$. Поэтому оператор $P_{3}$ является сглаживаюшим оператором в шкале $H_{0}^{s}$.

Теперь в формуле (3.7) для $P_{2} f$ можно произвести интегрирование по частям относительно $x^{\prime}$ достаточно большое количество раз. В результате получим

$$
P_{2}=P_{2}^{(N)}(\stackrel{1}{\hat{\xi}}, \stackrel{3}{2}, \hat{\xi})
$$

где символ $P_{2}^{(N)}\left(\xi^{\prime}, x, \xi\right)$ вместе с достаточно большим количеством производных убывает при $|\xi|+\left|\xi^{\prime}\right| \rightarrow \infty$ как $\left[|\xi|+\left|\xi^{\prime}\right|\right]^{-N}$ и число $N$ может быть сделано как угодно большим. Отсюда следует, что $P_{2}$ также является сглаживающим оператором.

Докажем, наконец, что $P_{1}$ является коническим псевдодифференциальным оператором порядка $2 m$. Для этого рассмотрим вектор-функцию $\delta S / \delta \xi\left(x ; \xi^{\prime}, \xi\right) \in \mathbb{R}^{n}$, у которой $i$-я компонента есть разностная производная

$$
\left(\frac{\delta S}{\delta \xi}\right)_{i} \stackrel{\text { def }}{\equiv} \frac{\delta S}{\delta \xi_{i}}\left(x ; \xi_{1}^{\prime}, \ldots, \xi_{i-1}^{\prime} ; \xi_{i}^{\prime}, \xi_{i} ; \xi_{i+1}, \ldots, \xi_{n}\right)
$$

Очевидно,

$$
S\left(x, \xi^{\prime}\right)-S(x, \xi)=\sum_{i}\left(\xi_{i}^{\prime}-\xi_{i}\right)\left(\frac{\delta S}{\delta \xi}\right)_{i}\left(x ; \xi^{\prime}, \xi\right)=\left(\xi^{\prime}-\xi\right) \frac{\delta S}{\delta \xi}\left(x ; \xi^{\prime}, \xi\right)
$$

Рассмотрим уравнение

$$
\widetilde{x}+\frac{\delta S}{\delta \xi}\left(\widetilde{x} ; \xi^{\prime}, \xi\right)=x
$$

для $\widetilde{x}$. Если якобиан

$$
J\left(\widetilde{x}, \xi^{\prime}, \xi\right)=\operatorname{det}\left(E+\frac{\partial}{\partial \widetilde{x}} \frac{\delta S}{\delta \xi}\left(\widetilde{x} ; \xi^{\prime}, \xi\right)\right)
$$

не равен нулю, то уравнение (3.8) определяет $\widetilde{x}$ как неявную функцию от переменных $x, \xi^{\prime}, \xi$ :

$$
\widetilde{x}=\widetilde{x}\left(x, \xi^{\prime}, \xi\right) .
$$


Заметим, что

$$
J\left(\widetilde{x}, \xi^{\prime}, \xi\right) \neq 0
$$

на $\operatorname{supp} \chi_{1}$ в силу того, что $\varepsilon$ - достаточно малое число, поскольку $J=J_{0} \neq 0$ на диагонали $\xi^{\prime}=\xi$ и оба якобиана суть однородные функции по импульсным переменным. Выберем число $\varepsilon$ так, чтобы условие (3.9) было удовлетворено. Тогда в формуле (3.7) для $P_{1} f$ можно сделать замену переменных $x^{\prime}=\widetilde{x}\left(y, \xi^{\prime}, \xi\right)$ во внутреннем интеграле. В результате получим

$$
\begin{aligned}
P_{1} f= & \left(\frac{1}{2 \pi}\right)^{2 n} \int e^{i\left(\xi x-\xi^{\prime} x^{\prime \prime}\right)} f\left(x^{\prime \prime}\right)\left\{\int e^{i y\left(\xi^{\prime}-\xi\right)}\left|J^{-1}\left(\widetilde{x}\left(y, \xi^{\prime}, \xi\right), \xi^{\prime}, \xi\right)\right|\right. \\
& \left.\times \varphi\left(\widetilde{x}\left(y, \xi^{\prime}, \xi\right), \xi^{\prime}\right) \bar{\varphi}\left(\widetilde{x}\left(y, \xi^{\prime}, \xi\right), \xi\right) \chi_{1}\left(\xi, \xi^{\prime}\right) d y\right\} d x^{\prime \prime} d \xi^{\prime} d \xi
\end{aligned}
$$

или

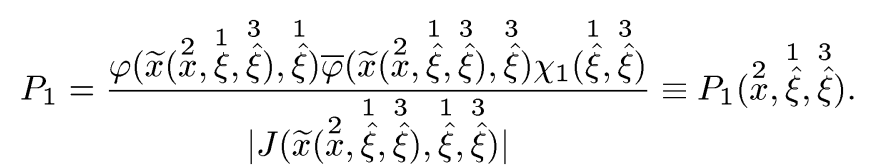

Теперь изучим зависимость символа $P_{1}\left(x, \xi^{\prime}, \xi\right)$ от $t$. Для этого перепишем систему (3.8) в виде

$$
\begin{gathered}
e^{-\widetilde{t}} \exp \left(-\frac{\delta \Phi}{\delta p}\left(e^{-\widetilde{t}}, \widetilde{\omega} ; \xi^{\prime}, \xi\right)\right)=e^{-t}, \\
\widetilde{\omega}+\frac{\delta \Phi}{\delta q}\left(e^{-\widetilde{t}}, \widetilde{\omega} ; \xi^{\prime}, \xi\right)=\omega
\end{gathered}
$$

или

$$
\begin{gathered}
\widetilde{r} \exp \left(-\frac{\delta \Phi}{\delta p}\left(\widetilde{r}, \widetilde{\omega} ; \xi^{\prime}, \xi\right)\right)=r, \\
\widetilde{\omega}+\frac{\delta \Phi}{\delta q}\left(\widetilde{r}, \widetilde{\omega} ; \xi^{\prime}, \xi\right)=\omega
\end{gathered}
$$

(напомним, что $\xi=(p, q)$ ).

Якобиан в левой части равенства относительно $(\widetilde{r}, \widetilde{\omega})$ есть

$$
J_{1}=\left|\begin{array}{cc}
\exp \left(-\frac{\delta \Phi}{\delta p}\right)\left(1-\widetilde{r} \frac{\partial}{\partial \widetilde{r}} \frac{\delta \Phi}{\delta p}\right) & -\widetilde{r} \frac{\partial}{\partial \widetilde{\omega}} \frac{\delta \Phi}{\delta p} \exp \left(-\frac{\delta \Phi}{\delta p}\right) \\
\frac{\partial}{\partial \widetilde{r}} \frac{\delta \Phi}{\delta q} & 1+\frac{\partial}{\partial \widetilde{\omega}} \frac{\delta \Phi}{\delta q}
\end{array}\right|
$$


С другой стороны,

$$
\begin{aligned}
& J=\left|\begin{array}{cc}
1-\widetilde{r} \frac{\partial}{\partial \widetilde{r}} \frac{\delta \Phi}{\delta p} & \frac{\partial}{\partial \widetilde{\omega}} \frac{\delta \Phi}{\delta p} \\
-\widetilde{r} \frac{\partial}{\partial \widetilde{r}} \frac{\delta \Phi}{\delta q} & 1+\frac{\partial}{\partial \widetilde{\omega}} \frac{\delta \Phi}{\delta q}
\end{array}\right| \\
& =\left|\begin{array}{cc}
1-\widetilde{r} \frac{\partial}{\partial \widetilde{r}} \frac{\delta \Phi}{\delta p} & \frac{\partial}{\partial \widetilde{\omega}} \frac{\delta \Phi}{\delta p} \\
\frac{\partial}{\partial \widetilde{r}} \frac{\delta \Phi}{\delta q} & -\widetilde{r}^{-1}\left(1+\frac{\partial}{\partial \widetilde{\omega}} \frac{\delta \Phi}{\delta q}\right)
\end{array}\right|(-\widetilde{r})^{n} \\
& =\left|\begin{array}{cc}
1-\widetilde{r} \frac{\partial}{\partial \widetilde{r}} \frac{\delta \Phi}{\delta p} & -\widetilde{r} \frac{\partial}{\partial \widetilde{\omega}} \frac{\delta \Phi}{\delta p} \\
\frac{\partial}{\partial \widetilde{r}} \frac{\delta \Phi}{\delta q} & 1+\frac{\partial}{\partial \widetilde{\omega}} \frac{\delta \Phi}{\delta q}
\end{array}\right|=\exp \left(n \frac{\delta \Phi}{\delta p}\right) J_{1} .
\end{aligned}
$$

Отсюда следует, что $\widetilde{r}=e^{-\widetilde{t}}$ является гладкой функцией переменных $r=e^{-t}$ и $\omega$ и

$$
P_{1}(\stackrel{2}{x}, \stackrel{3}{\xi}, \stackrel{\hat{\xi}}{\xi})=Q\left(e^{2}-t, \stackrel{2}{\omega},-i \frac{1}{\partial t},-i \frac{\stackrel{\partial}{\partial}}{\partial \omega},-i \frac{3}{\partial t},-i \frac{3}{\partial \omega}\right)
$$

где $Q\left(r, \omega, \xi^{\prime}, \xi\right)$ - гладкая функщия, асимптотически однородная порядка $2 m$ по переменным $\left(\xi, \xi^{\prime}\right)$. (Якобиан, как и функция $\chi$, суть однородная функция нулевого порядка.) Таким образом, оператор $P_{1}$ есть конический псевдодифференциальный оператор в шкале $H_{0}^{s}(M)$. Главный символ псевдодифференциального оператора $T^{*} T$ получается, если положить $\xi^{\prime}=\xi$ в формуле для символа $P_{1}$ и взять главную часть относительно однородности. Соответствующие вычисления мы позволим себе опустить. Доказательство леммы 1.3 завершено.

\section{Список литературы}

1. Weinstein A. Fourier integral operators, quantization, and the spectrum of a riemannian manifold // Géométrie Symplectique et Physique Mathématique. Colloque Internationale de Centre National de la Recherche Scientifique. 1976. V. 237. P. 289-298.

2. Арнольд В. И. Математические методы классической механики. М.: Наука, 1989.

3. Guillemin V. W. Toeplitz operators in $n$-dimensions // Integral Equations and Operator Theory. 1984. V. 7. P. 145-205.

4. Epstein C., Melrose R. Shrinking tubes and the $\delta$-Neumann problem. Preprint, 1997.

5. Weinstein $A$. Some questions about the index of quantized contact transformations // RIMS Kôkûryuku. 1997. V. 104. P. 1-14.

6. Epstein C., Melrose R. Contact degree and the index of Fourier integral operators // Math. Res. Lett. 1998. V. 5. № 3. P. 363-381.

7. Schulze B.- $W$. Pseudodifferential Operators on Manifolds with Singularities. Amsterdam: North-Holland, 1991.

8. Nazaikinskii V., Schulze B.-W., Sternin B. The Index of Quantized Contact Transformations on Manifolds with Conical Singularities. Preprint № 98/16. Univ. Potsdam, Institut für Mathematik Potsdam, August 1998.

9. Назайкинский В. Е., Стернин Б. Ю., Шульще Б.-В. Индекс квантованных контактных преобразований на многообразиях с коническими особенностями // Докл. РАН. 1999. Т. 368. № 5. С. 598-600. 
10. Schulze B.-W., Sternin B., Shatalov V. Differential Equations on Singular Manifolds. Semiclassical Theory and Operator Algebras. V. 15. Of Mathematics Topics. Berlin-N.Y.: Wiley-VCH Verlag, 1998.

11. Nazaikinskii V., Schulze B.-W., Sternin B., Shatalov V. Quantization of Symplectic Transformations on Manifolds with Conical Singularities. Preprint № 97/23. Univ. Potsdam, Institut für Mathematik, Potsdam, July 1997.

12. Melrose R. Transformation of boundary problems // Acta Math. 1981. V. 147. P. 149-236.

13. Nazaikinskii V., Sternin B., Shatalov V. Contact Geometry and Linear Differential Equations. De Gruyter Expositions in Mathematics. Berlin-N. Y.: Walter de Gruyter Publishers, 1992.

14. Mishchenko A., Shatalov V., Sternin B. Lagrangian Manifolds and the Maslov Operator. Berlin-Heidelberg: Springer-Verlag, 1990.

15. Маслов В. П. Теория возмущений и асимптотические методы. М.: Изд-во МГУ, 1965.

16. Назайкинский В. Е., Ошмян В.Г., Стернин Б. Ю., Шаталов В.Е. Интегральные операторы Фурье и канонический оператор // УМН. 1981. Т. 36. № 2. С. 81-140.

17. Schulze B.-W., Sternin B., Shatalov V. Structure rings of singularities and differential equations // Differential Equations, Asymptotic Analysis, and Mathematical Physics, number 100 in Mathematical Research. Berlin: Akademie Verlag, 1996. P. 325-347.

18. Schulze B.-W. Boundary Value Problems and Singular Pseudo-Differential Operators. Chichester: J. Wiley, 1997.

19. Schulze B.-W., Sternin B., Shatalov V. On the index of differential operators on manifolds with conical singularities // Annals of Global Analysis and Geometry. 1998. V. 16. № 2. P. 141-172.

20. Fedosov B. V. Deformation Quantization and Index Theory. V. 9. Of Mathematical Topics. Berlin: Akademie Verlag, 1996.

21. Nazaikinskii V., Schulze B.-W., Sternin B., Shatalov V. The Atiyah-Bott-Lefschetz fixed point theorem for manifolds with conical singularities // Annals of Global Analysis and Geometry. 1999. V. 17. № 5. P. 409-439.

22. Nazaikinskii V., Sternin B., Shatalov V. Methods of Noncommutative Analysis. Theory and Applications. Mathematical Studies. Berlin-N.Y.: Walter de Gruyter Publishers, 1995.

23. Маслов В. П. Операторные методы. М.: Наука, 1973.

Москва, Потсдам

Поступило в редакцию 30.X.1998 